\title{
Aeroacoustic tunnel effect in noise shielding problems
}

\author{
Jan W. Delfs* \\ DLR - Deutsches Zentrum für Luft- und Raumfahrt e.V. \\ Institute of Aerodynamics and Flow Technology, Department Technical Acoustics \\ 38108 Braunschweig, Germany
}

\begin{abstract}
The question is addressed, under which conditions the commonly applied neglect of shear in the flow past an acoustically shielding object may not be justified, even at low Mach numbers, representative for take-off and landing of aircraft. The numerical results generated to quantify the engine noise shielding effect of a high lift airfoil in the EU project NACRE are taken as starting point. These results showed an unexpectedly significant influence of shear on the shielding when compared to a simplified approach neglecting the shear at a flow Mach number of $M=0.3$. The paper gives an explanation of this phenomenon based on total reflection and accompanying evanescent waves in the shear flow near the flap slot. The problem is stripped down to the essential physical ingredients and is then reproduced in a much more simplified setup. The investigation shows that evanescent waves below a reflecting shear layer may become propagational in the presence of an edge or slot, i.e. sound may "tunnel" through even a totally reflecting shearlayer and radiate into the acoustic shadow zones predicted with simple methods, which neglect shear.
\end{abstract}

\section{Introduction}

For the purpose of predicting scattering of engine sound at aircraft geometries at take-off and landing, i.e. for low Mach number flow it is common practice to neglect viscous flow effects or even the flow alltogether.

*Head of Department and Professor, AIAA Senior Member.
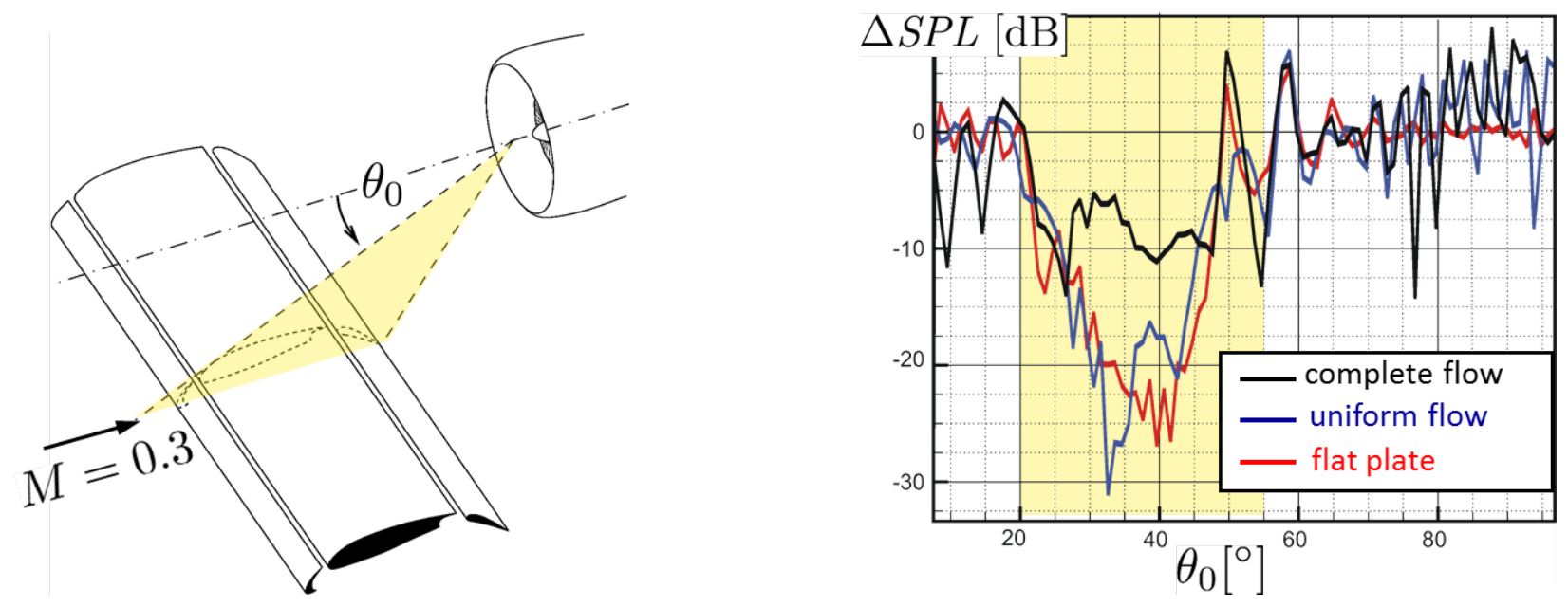

Figure 1. Numerical simulation of engine sound shielding at high lift system. Left: sketch of configuration, right: (see ${ }^{5}$ ) shielding results for complete (viscous) flow, uniform flow, and a flat plate of similar dimensions and uniform flow; incident sound signal represents the sum of the first 4 azimuthal modes, radial mode order zero, $f=11.733 \mathrm{kHz}$, chord length of retracted airfoil $c=0.543 \mathbf{m}$. 
Not having to deal with any boundary or shear flow is highly attractive from a computational point of view, because then very efficient methods like the Fast Multipole Boundary Element Method may be used to predict the acoustic installation effects of full scale geometries in the complete audible frequency range of interest. Computational approaches were successful to incoporate potential flow effects at small Mach

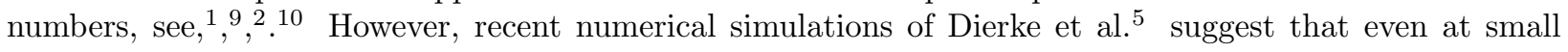
Mach numbers the neglect of viscous flow effects may lead to very large errors in the prediction of engine fan sound shielding at a high lift wing. For certain conditions level differences on the order of $10 \mathrm{~dB}$ were observed when viscous effects in the mean flow field were not taken into account. Even worse, the results consistently showed a respective overprediction of beneficial sound shielding when using an inviscid flow field. The paper offers a physical explanation for the observed phenomena, based on evanescent waves and some sort of "tunnel effect". The question is discussed under which conditions the neglect of viscous flow effects may lead to broadly wrong solutions for the prediction of acoustic installation problems, even at low subsonic Mach number flows.

\section{Hypothesis and proof of concept}

The hypothesis is that the strong viscous (shear) flow effect observed for certain engine modes in the shielding problem discussed in ${ }^{5}$ is due to edge scattering of evanescent (non-propagational) pressure waves into propagational pressure. This phenomenon occurs whenever sound waves hit the scattering object at shallow angles $\theta_{i}<\theta_{i}^{\text {tot }}$ against the flow direction. In this context "shallow" means incidence angles below the critical angle of specular or total reflection $\theta_{i}^{\text {tot }}$. The phenomenon has similarities with those found at a double prism for light or microwaves and is called "frustrated total reflection" 7

Sound traveling through a shearlayer experiences refraction. A plane sinusoidal wave with a pressure amplitude of unity is considered. It has a phase normal inclined by the angle $\vartheta_{i}$ with respect to the flow (and shearlayer) direction. The corresponding ray travels along the true propagation angle $\vartheta_{0}$ as sketched in the upper part of figure 2 The angles $\theta$ mentioned in figure 1 relate like $\theta=\pi-\vartheta$. At the shearlayer a part of the wave is reflected under a angle $\pi-\vartheta_{i}$ while another part is transmitted across the layer, experiencing a characteristic change in propagation direction $\vartheta_{t}$ representing simultaneaously the true propagation because here the medium is at rest. The lower part of the figure depicts the refraction at different wave incidence angles $\vartheta_{i}=45^{\circ}, 135^{\circ}$ and at $\vartheta_{i}=\vartheta_{i}^{\text {tot }}+10^{\circ}$, where the critical incidence of total reflection is $\vartheta_{i}^{\text {tot }}=\cos ^{-1}(-1 /(1+M))$. Beyond the ciritcal angle total reflection occurs, i.e. the incoming
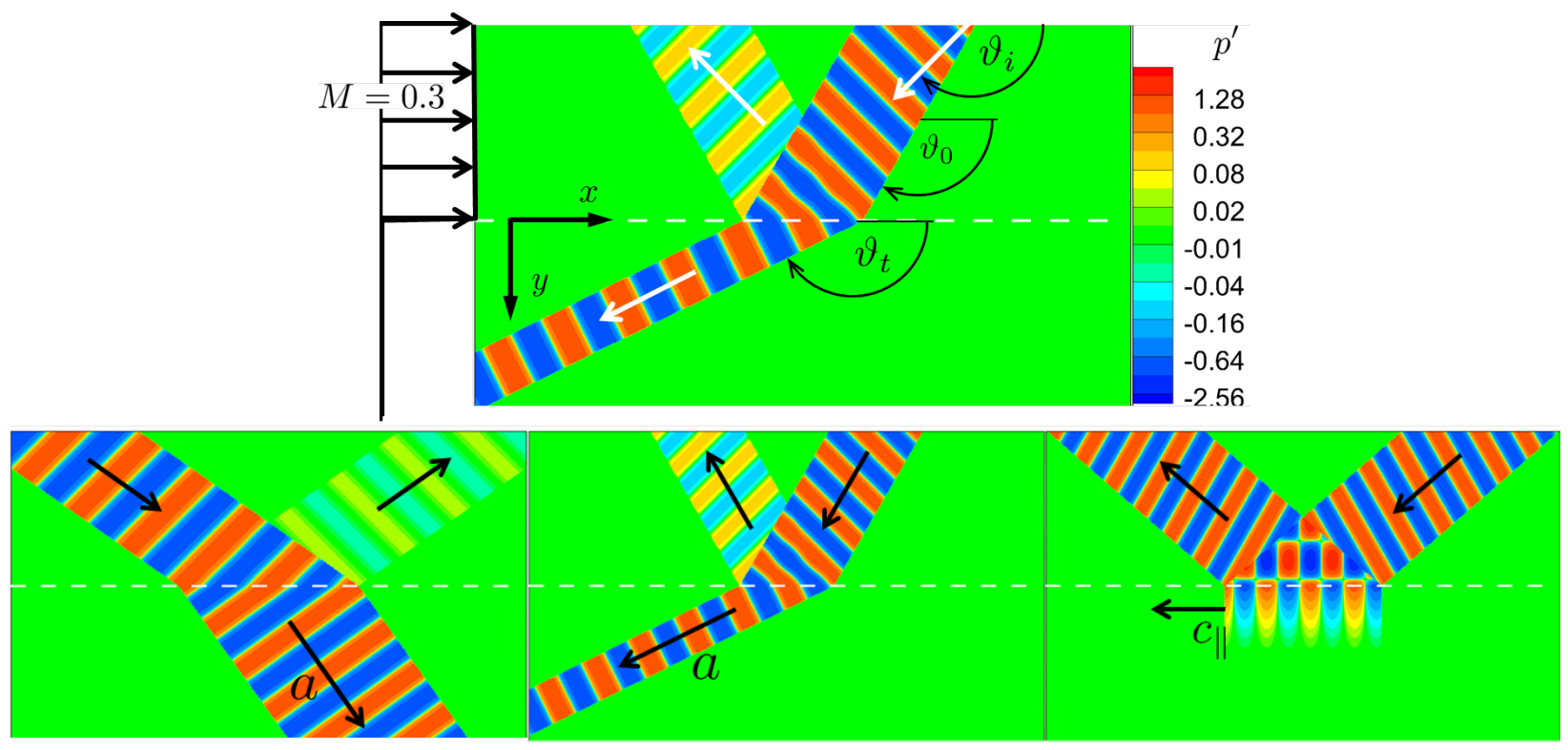

Figure 2. Upper figure: definition of refraction problem at infinitely thin shearlayer; lower part: left: incidence angle $\vartheta_{i}=45^{\circ}$, centre: $\vartheta_{i}=135^{\circ}$, right: $\vartheta_{i}=\vartheta_{i}^{\text {tot }}+10^{\circ}$. 
wave is completely reflected off the shearlayer, similar as at a hard wall or as may be observed for light at the interface between glass and air of a prisma. Below the shearlayer, i.e. in the medium at rest, one observes so called evanescent waves, which travel in upstream direction at a speed $c_{\|}=a\left|M+1 / \cos \vartheta_{i}\right|$ smaller than the speed of sound $a$. In that sense they do not represent sound waves. The exponential decay of the evanescent waves in the direction normal to the shearlayer increases with frequency and the angle of incidence, and it decreases with the flow Mach number.

In order to decide whether or not evanescent waves may influence the mentioned shielding problem it is helpful to take a look at some details of the geometry and flow. According to figure 1 the configuration studied in 5 featured a rear mounted turbofan; i.e. forward fan sound waves hit the high lift wing at shallow angles against the flow direction. Hence, at least locally the setup features a situation as characterized by the lower two pictures in the right of figure 2. An estimate of the local incidence angles is shown in figure 3 The angle of incidence relates to the orientation of the boundary layers adjacent to the wing's upper surface. Near the surface the flow orientation (and thus the boundary layer) is estimated to be about tangential to the surface with a strong shear normal to it. In the picture it is crudely approxiated by a dashed straight line. The arrow shown represents the direction of the sound ray, along which the wave with the critical angle

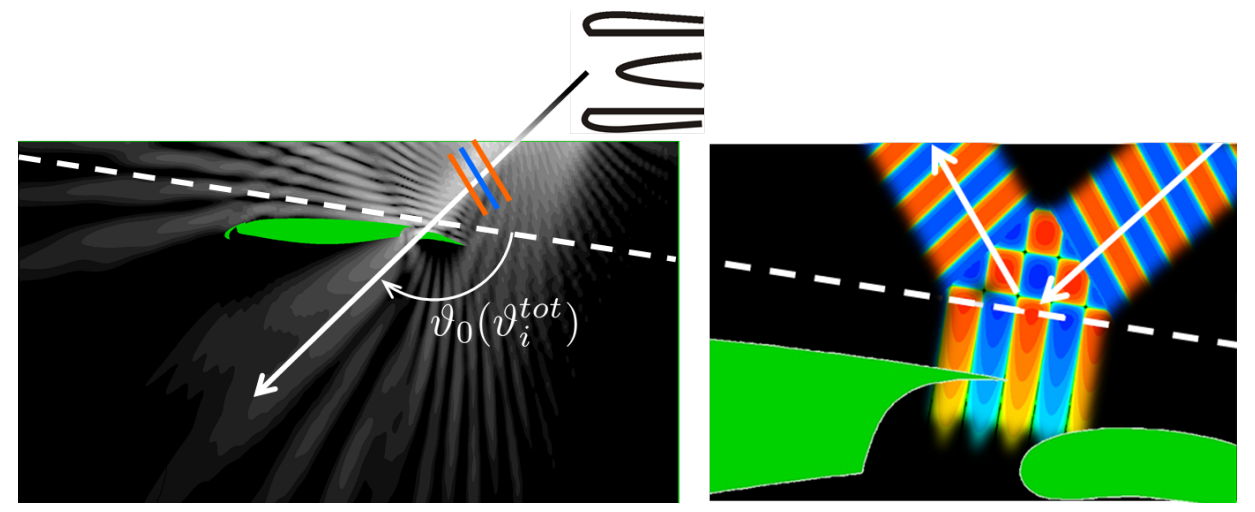

Figure 3. Orientation of critical ray experiencing total reflection (data taken from ${ }^{5}$ represent SPL in grayscale), right: sketch of totally reflected ray with evanescent waves below Mach0.3 plane shearlayer for $\vartheta_{i}=\vartheta_{i}^{\text {tot }}+2^{\circ}$.

of incidence $\vartheta_{i}^{\text {crit }}$ (related to the dashed line) travels for an assumed constant flow of Mach number $M=0.3$. This simple geometric consideration reveals that the orientation of critical incidence (beyond which total reflection at the shear layer occurs) happens to coincide with the line between the center of the nacelle inlet plane and the position on the flap, slightly downstream of the gap between main element and flap. Hence, with the hypothesis in mind that an interaction of an evanescent wave with an edge (present in the gap between main element and flap) may cause the generation of sound, it appears reasonable to continue the analysis. It is noted, that this geometric constellation also means that the whole main element including the slat are subject to sound incident under angles well beyond the critical angle, such that one may assume that in this region the shielding is entirely according to total reflection at the boundary layer. This effect is further augmented by the curvature of the geometry.

It is more simple to study the problem in two instead of three dimensions. Moreover, the sound source used in the mentioned NACRE case study was a complex 3D fan tone sound field. Therefore in a first step the same geometry was re-calculated using a $2 \mathrm{D}$ point source of unit strength $\hat{\theta}_{p}$ (see appendix A located at the corresponding position of the engine intake centre from the 3D NACRE case. Figure 4 shows the result of a numerical solution of the Linearized Euler Equations (LEE) obtained with DLR's PIANO finte difference CAA code ${ }^{3}$ A frequency of $f=10 \mathrm{kHz}$ was considered for the high lift airfoil with a chord length of $c=0.543 \mathrm{~m}$ at a freestream Mach number of $M=0.3$. A multiblock grid with about 160k points was used, which resulted in a wave resolution of about 7 points per wavelength in the upstream direction. Also the 2D NACRE case shows the effect observed in 3D, namely that there are higher sound pressure amplitudes in the shadow zone with viscous as opposed to constant mean flow. Compared to the 3D NACRE case the viscous flow effect is somewhat smaller. It remains to be studied, whether this is a general effect. The study shows that it is useful to continue the study in a simplified form. 

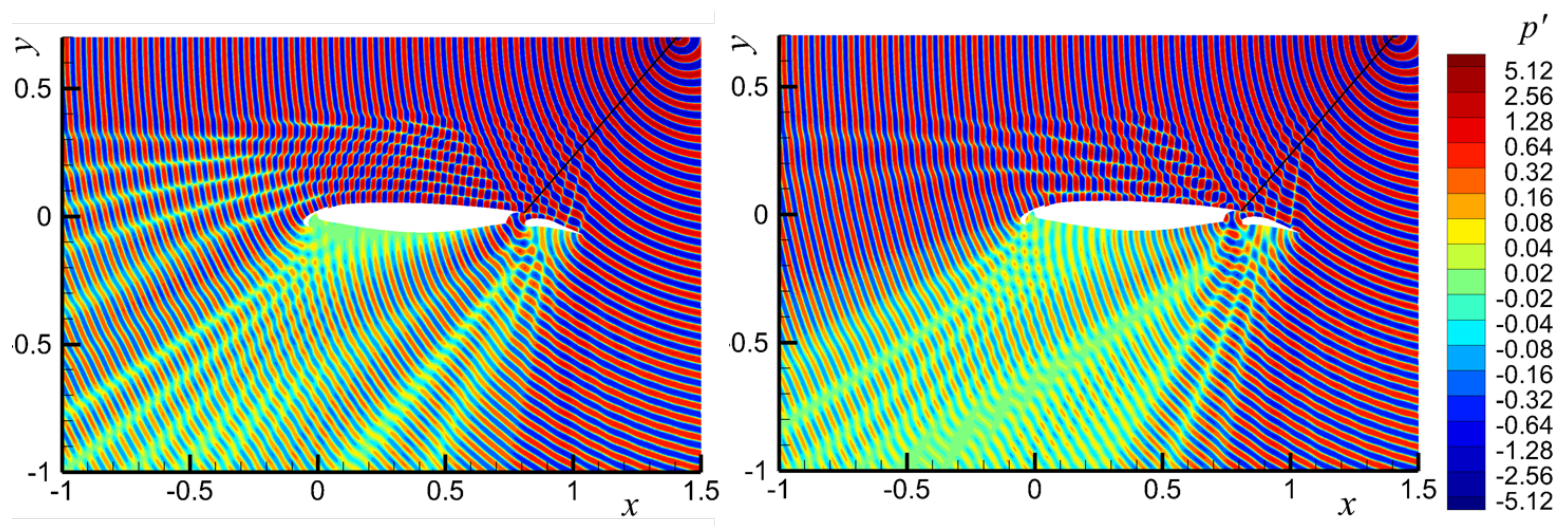

Figure 4. Comparison of NACRE shielding problem in 2D with a point source. Snapshot of pressure fluctuation $p^{\prime}$ for viscous mean flow (left) and constant mean flow (right). The black line shows the direction, under which total reflection occurs for a boundary layer oriented parallel to the contour of the main element near it's trailing edge.

\section{Computational study and Results}

In order to investigate whether the edges of the gap slot would in principle be able to generate sound of appreciable magnitude in this first study the problem is highly simplified and reduced to the most essential elements. The purpose of this simplification is to gain more general understanding of the physics involved.

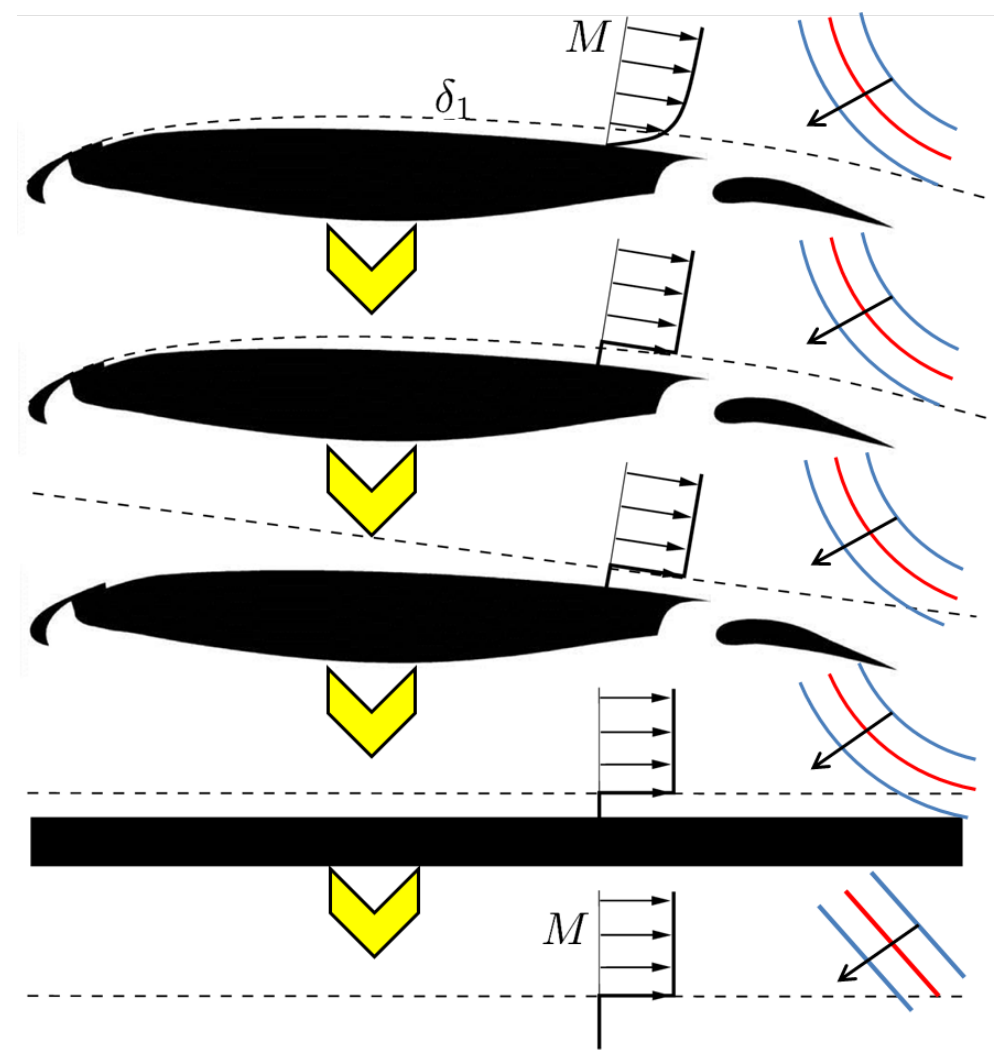

Figure 5. Simplification of original problem. top to bottom: a) original problem, b) reduced to the main acoustic effect of the shear (refraction of sound), c) reduced to local geometric / indicence angles from the region of interest, d) reduction to plane homogeneous problem, e) reduced to the conditions relevant for the generation of the evanescent waves. In a later stage, geometry elements with edges will be re-introduced below the shearlayer (see below). 
Figure 5 visualizes this drastic simplification. The top picture shows the original problem with a dashed line representing some boundary layer characteristic, here the displacement thickness $\delta_{1}$. As far as the interaction of the system with incident sound waves is concerned the essential ingredient is the shear in a quasi-parallel layer as presented by the bounday layer over the suction side of the high lift system. Therefore is seems justified to replace the actual boundary layer profile by a jump layer (vortex sheet) at the distance to the surface of about the displacement thickness $\delta_{1}$. For capturing the main effects it should be suffient to focus on the flow / sound incidence situation in the vicinity of the flap slot area and thus the neglect of the shearlayer curvature (the radius of which is very large compared to the incident acoustic wavelengths anyways). Assuming the occurrence of evanescent waves to be the key feature of the mechanism studied, it is sufficient to look at a geometrically homogeneous problem first, which further reduces to considering plane waves hitting a free planar shearlayer of zero thickness as the most simple starting point for the essence of the problem. With this setup the entire plane wave field including the evanescent waves may be described easily and analytically. For this purpose a co-ordinate system is introduced as shown in 2 . If the pressure field $p^{\prime}(x, y, t)$ is described as

$$
\begin{aligned}
& p^{\prime}(x, y, t)=p^{-}(y) \exp (i \omega t-i \alpha x) \text { for } \quad y<0 \\
& p^{\prime}(x, y, t)=p^{+}(y) \exp (i \omega t-i \alpha x) \text { for } \quad y>0
\end{aligned}
$$

with $\alpha=k_{-\infty} \cos \vartheta_{i}$ and $k_{-\infty}=k /\left(1+M \cos \vartheta_{i}\right)$ while $k=\omega / a$ for an incident wave under orientaion $\vartheta_{i}$ with angular frequency $\omega$, then the derivation of $p^{ \pm}$is a matter of textbook material (see. e.g. Delfs ${ }^{4}$ ). One obtains

$$
p^{-}(y)=p^{i} \exp \left(-i\left|k_{-\infty} \sin \vartheta_{i}\right| y\right)+p^{r} \exp \left(i\left|k_{\infty} \sin \vartheta_{i}\right| y\right)
$$

with $p^{i}$ the amplitude of the incoming wave and $p^{r}$ the amplitude of the reflected wave. For the pressure distribution of the outgoing or evanescent wave, i.e. on the side $y>0$ the expression reads

$$
p^{+}(y)=p^{t} \exp \left(-i k_{-\infty}[H(\sigma)+i\{1-H(\sigma)\}] \sqrt{|\sigma|} y\right)
$$

with $\sigma=\cos ^{2} \vartheta_{i}-\left(1+M \cos \vartheta_{i}\right)^{2}$ and $H(\sigma)$ the Heaviside function. The amplitude of the reflected and transmitted (evanescent) wave is

$$
\begin{aligned}
\frac{p^{r}}{p^{i}} & =\frac{\sin \vartheta_{i}\left(1+M \cos \vartheta_{i}\right)^{2}-\sqrt{|\sigma|}(1-H(\sigma))+i \sqrt{|\sigma|} H(\sigma)}{\sin \vartheta_{i}\left(1+M \cos \vartheta_{i}\right)^{2}+\sqrt{|\sigma|}(1-H(\sigma))-i \sqrt{|\sigma|} H(\sigma)} \\
\frac{p^{t}}{p^{i}} & =\frac{2 \sin \vartheta_{i}\left(1+M \cos \vartheta_{i}\right)^{2}}{\sin \vartheta_{i}\left(1+M \cos \vartheta_{i}\right)^{2}+\sqrt{|\sigma|}(1-H(\sigma))-i \sqrt{|\sigma|} H(\sigma)}
\end{aligned}
$$

For the study of edge sound generation in the area of evanescent waves a simplified approach was taken in this first study. Since small Mach number flows are considered a simple acoustic inhouse boundary element method was used to compute the sound field generated by a plate placed inside the domain below the shearlayer with no flow. The analytically known evanescent pressure field was specified at the surface elements as an input to the BEM, which generated the corresponding acoustic response in order to satisfy the hard wall condition. All simulations were done using a resolution with more than 8 elements per wavelength. The amplitude of the incoming wave $p^{i}$ was set equal to unity for all simulations.

\section{A. Shear flow effects at simple plate geometry - infinitely thin layer}

The upper left contour plot of figure 6 depicts the analytical solution (1.6) for $M=0.3$ at $f=500 \mathrm{~Hz}$ at an angle of incidence of $\vartheta_{i}=\vartheta_{i}^{\text {tot }}+10^{\circ}$. For the other pictures in the figure a plate of length $l=0.5 \mathrm{~m}$ and a thickness of $t=0.02 \mathrm{~m}$ was placed right below the shearlayer (distance $d=0.03 \mathrm{~m}$ ). The plate had an inclination of $45^{\circ}$ with respect to the flow direction mimicing the acoustic effect of a deployed flap. Clearly, this figure shows a classical sound generation at the shearlayer side edge of the plate. The plate scatters the evanescent pressure into sound which is primarily radiated towards the upstream and downward directions. This is the sector which is particularly relevant as far as the shielding problem at the original high lift system is concerned since this is the direction of the shadow zone. The variation of the angle of incidence to a value $\vartheta_{i}=\vartheta_{i}^{\text {tot }}+5^{\circ}$ reveals a slightly stronger scattered wave but features an otherwise similar wave pattern. Also the variation of the flow Mach number from $M=0.3$ to $M=0.2$ in the lower right part of figure 6 displays 


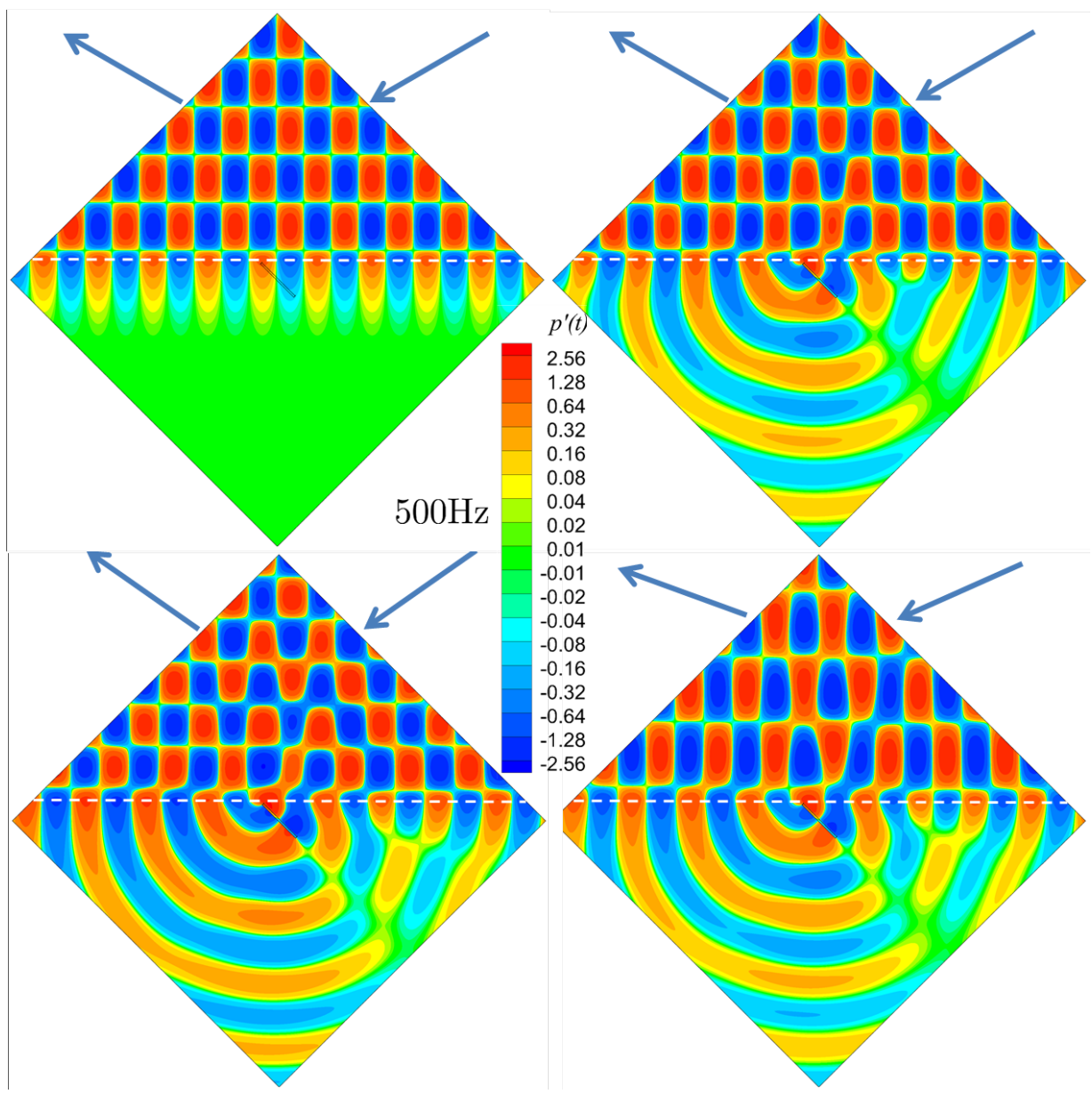

Figure 6. Scattering of evanescent waves below $M=0.3$ shearlayer at $\vartheta_{i}=\vartheta_{i}^{\text {tot }}+10^{\circ}$. Flow from left to right above dashed line, arrows indicating wave orientation $\vartheta_{i}$. Upper left: no scattering object, upper right: scattering plate in center, inclined by $45^{\circ}$, lower left: decrease of angle of incidence by $\Delta \vartheta_{i}=5^{\circ}$, lower right: decrease of Mach number to $M=0.2$

no large changes, i.e. the shown effect is quite stable under variations. In particular in an even smaller Mach number flow significant sound generation due to the edge scattering is present.

Figure 7 depicts variations of the frequency from $500 \mathrm{~Hz}$ to $1000 \mathrm{~Hz}$ as well as different plate lengths and orientations. The angle of indidence is fixed to $\vartheta_{i}=\vartheta_{i}^{\text {tot }}+2^{\circ}$, i.e. quite near to the critical angle of incidence. This case is closer to the original problem of the high lift wing, where the flap slot edges are located at about an incidence angle of the choosen value (see figure 3). Sound generation due to scattering is expected to be high in this regime since the spatial decay of the evanescent waves is low near the critical angle. The upper left diagram of figure 7 shows a plate of length $l=1.5 \mathrm{~m}$ placed parallel below the shearlayer again at the same distance of $d=0.03 \mathrm{~m}$. A frequency of $f=1000 \mathrm{~Hz}$ is considered. Again considerable sound is generated through the tunnel effect of the plate. In fact, the larger dimension of the plate in combination with the higher frequency reveals that the sound is generated entirely at the leading and trailing edges of the plate. Also, the maximium amplitudes are again radiated in the shallow upstream direction. If the long plate is inclined by $10^{\circ}$ the sound radiation remains similar in so far as again the main radiation is directed in the shallow upstream direction.

\section{B. Shear flow effects at slotted plate geometry - infinitely thin layer}

In order to represent the shielding situation of a slotted high lift system more realistically a slotted plate arrangement was considered next. The geometry is defined as in sketch 8 . In order to keep the case as simple as possible again plane waves were considered, impinging under a defined angle slightly beyond the critical 

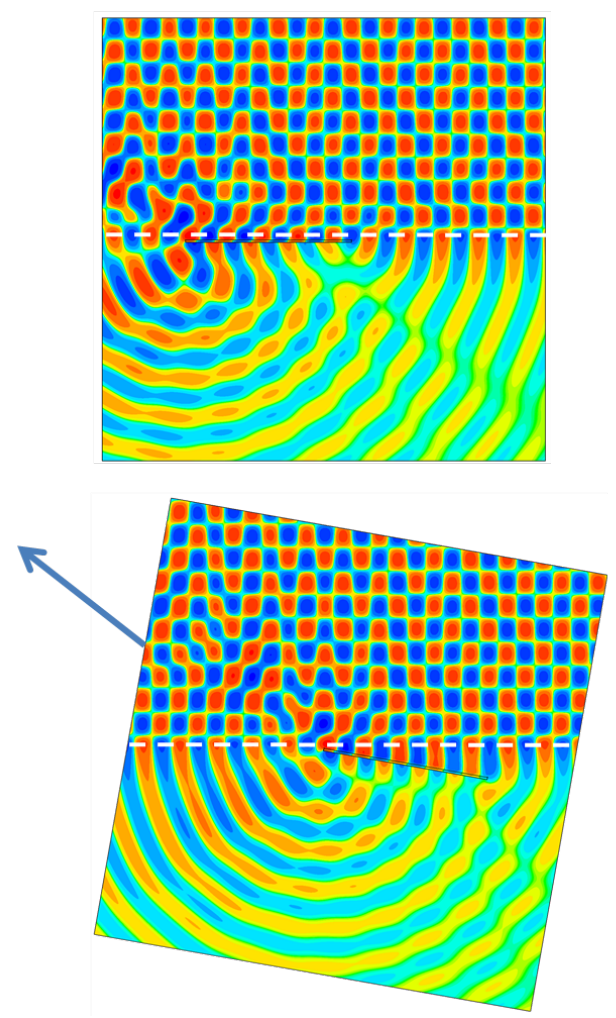
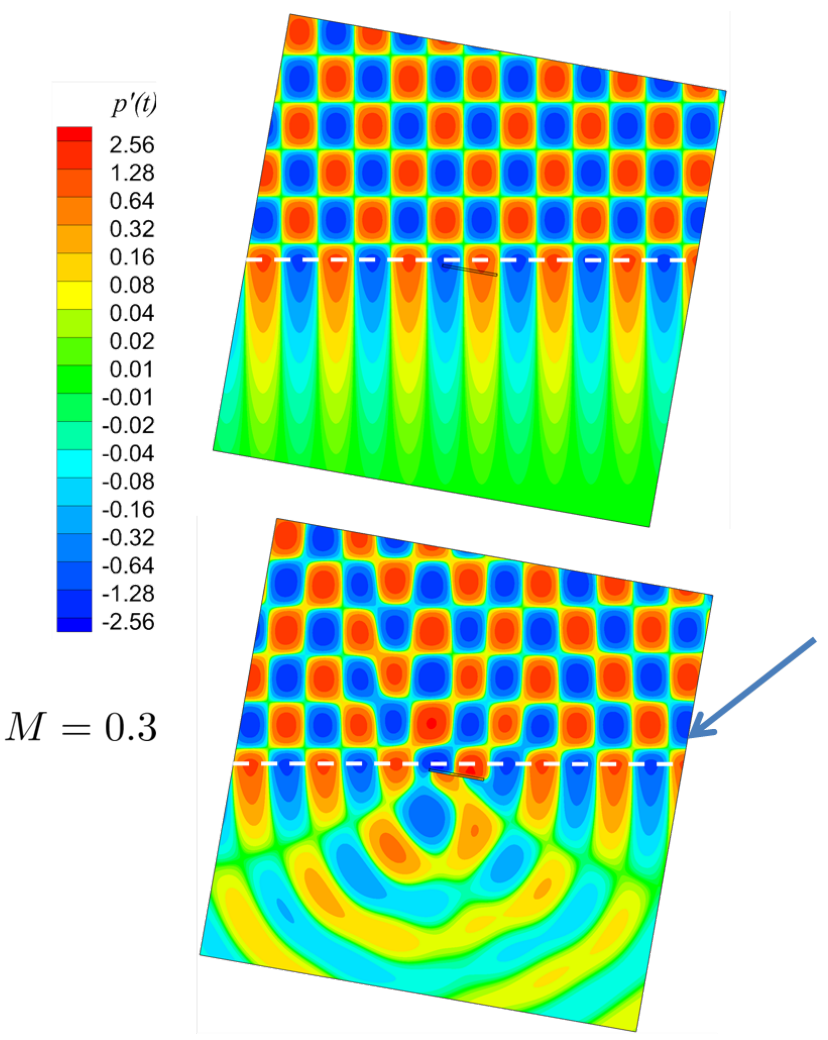

Figure 7. Scattering of evanescent waves below $M=0.3$ shearlayer at $\vartheta_{i}=\vartheta_{i}^{\text {tot }}+2^{\circ}$. Flow from left to right above dashed line, arrows indicating wave orientation $\vartheta_{i}$. Right column: top shows evanescent field for frequency of $500 \mathrm{~Hz}$ (no plate), bottom shows the effect of a plate of length $l=0.5 \mathrm{~m}$ at a small inclination angle of $10^{\circ}$; left column: frequency of $1000 \mathrm{~Hz}$, long plate $l=1.5 \mathrm{~m}$. top shows sound generation for parallel orientation, bottom for inclination of $10^{\circ}$.

angle of total reflection onto the plate arrangement. Using the same BEM method as in the previous section several variations were made on the slotted plate arrangement. The influence of the slot width, the distance of the shear layer to the plate, the angle of incidence of the plane wave and the frequency were studied. The results obtained for the case of a shearlayer were then compared against the same situation with a constant uniform flow all over the domain. The BEM boundary integral formulation for the convective wave equation is restricted to contain only linear terms in the Mach number; also slender geometries are assumed, certainly valid for the plate arrangement with a relative thickness of about $1.3 \%$. For these assumptions the actual boundary integral to be solved is obtained simply by replacing the free-field Green's function for the standard wave equation to the one for the convective wave equation. One has to bear in mind that the BEM simulation for the plate under the influence of a shearlayer assumes no flow, which is correct only in the domain below the shearlayer (which of course is at the same time the most interesting area in view of the shielding problem). In contrast the simulation results with uniform flow are valid in the entire domain.

Before turning to these various parameter variations one has to find a way to isolate the effect of the slot for the cases with and without shearlayer. The studies in the previous section had shown already the importance of the leading and trailing edge of the plate. When considering a plate arrangement of finite chord length $l_{c}$ the scattered sound field will always be a complex superposition of the effects of edges and the slot, which will make it difficult to assess the importance of the slot, see for instance figure 9. Clearly, it is hard to understand the role of the slot in generating these complex patterns. Therefore in order to isolate the slot effect the pressure field of the unslotted plate of equal length $l_{c}$ and computed subject to exactly the same conditions is subtracted from the pressure field found for the slotted plate. This subtraction has to be carried out at equal phase of the source. Given the linearity of the problem, especially the independence of the source from the resulting sound field, in this way the scattering of the incident field at the leading and trailing edge of the plate may be removed from the overall field and the remaining field can be attributed to 


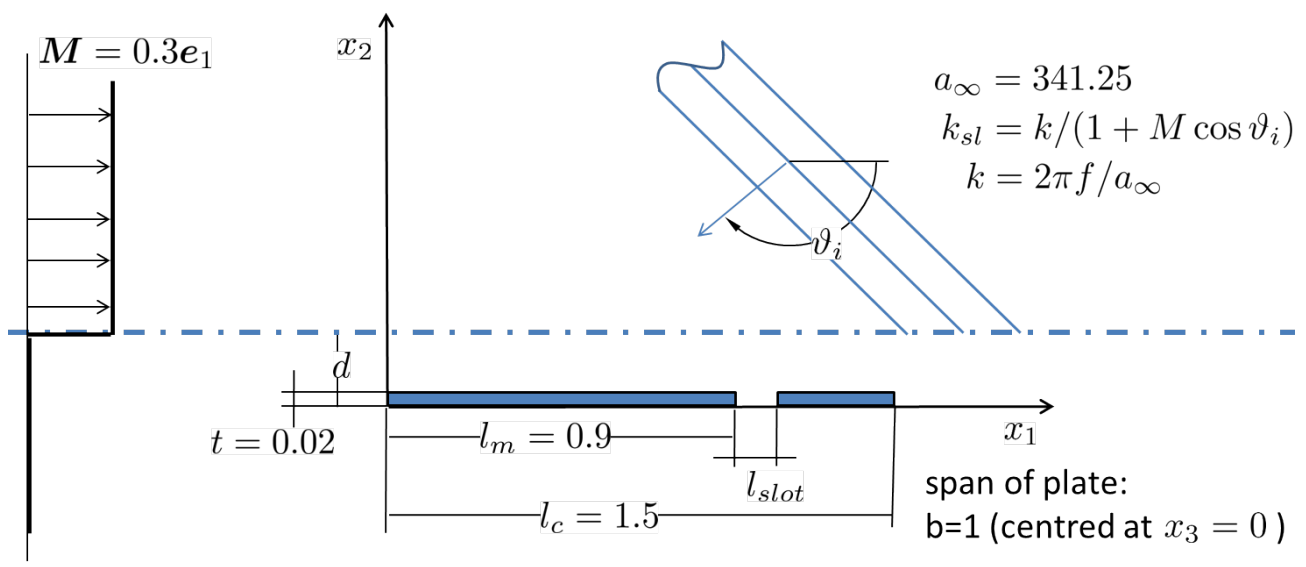

Figure 8. Case study on slotted plate. All quantities are given in SI units. The frequency of the plane incident wave is denoted with $f$.

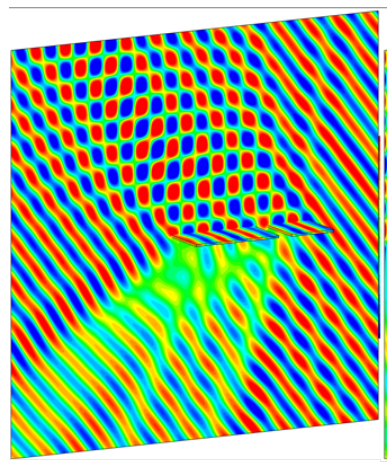

uniform flow

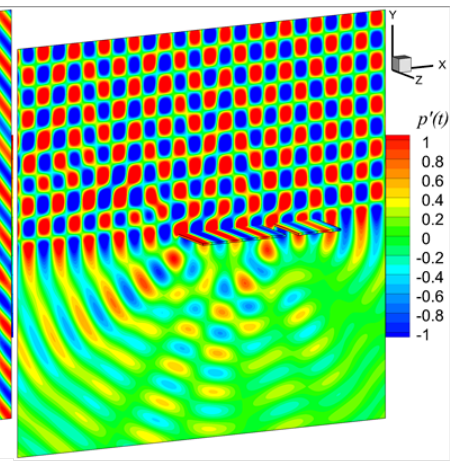

shear layer

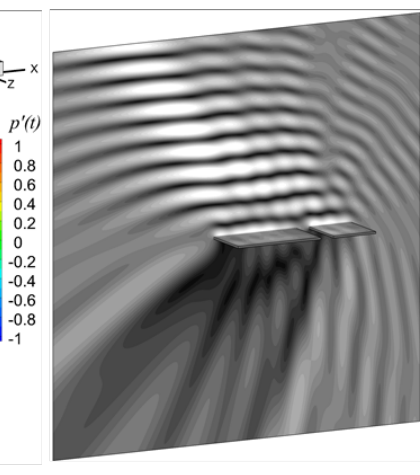

uniform flow

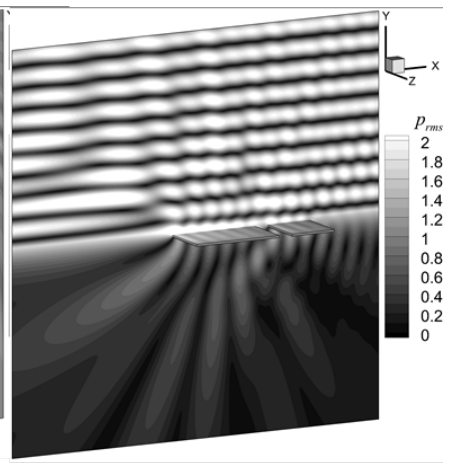

shear layer

Figure 9. Pressure field for $l_{\text {slot }}=9.4 \% l_{c}, f=1000 \mathrm{~Hz}, \vartheta_{i}=\vartheta_{i}^{t o t}+2^{\circ}$ for slotted plate (snapshot in color, rms-values in greyscale) in case of shearlayer at $d=0.058 \mathrm{~m}$ and for uniform flow.

the slot. The diagrams to follow therefore show either the difference field

$$
\Delta p^{\prime}=p_{\text {slotted }}^{\prime}(\boldsymbol{x}, t)-p_{\text {unslotted }}^{\prime}(\boldsymbol{x}, t)
$$

or the magnitude of it in the form of a level

$$
\Delta L=20 \lg \left(\Delta p_{\text {rms }}\right)+\text { const }
$$

with an arbitrary but fixed constant const.

The effect of the slotwidth for the case of the plate below a shearlayer is depicted in figure 10. In a shapshot the difference plots (upper row in figure) show that the signal amplitudes increase with increasing slotwidth. Sound is observed to emanate from the slot region; and due to the non-compactness of the object a multi lobed radiation pattern forms. As observed earlier, the sound field generated by the slot in response to the evanescent pressure field below the shearlayer tends to radiate more sound upstream, especially as the slot widens. The results of the same variation of the slot width is shown in figure 11 for the uniform flow case. Starting from the most wide slot at $l_{\text {slot }}=18.4 \% l_{c}$ one observes the same general trend as seen for the plate with the shear flow. As the slot narrows the pressure amplitudes decrease. This effect appears to be much stronger than in the case of the shear flow. For the large gap part of the sound directly propagates through the opening as the wavelength is no longer large compared to the slot width. On the other hand, the more the gap narrows, the lower the sound field transmitted through the slot because this happens through a "inefficient" diffraction process. This situation is in contrast to the situation seen for the slot subject to 


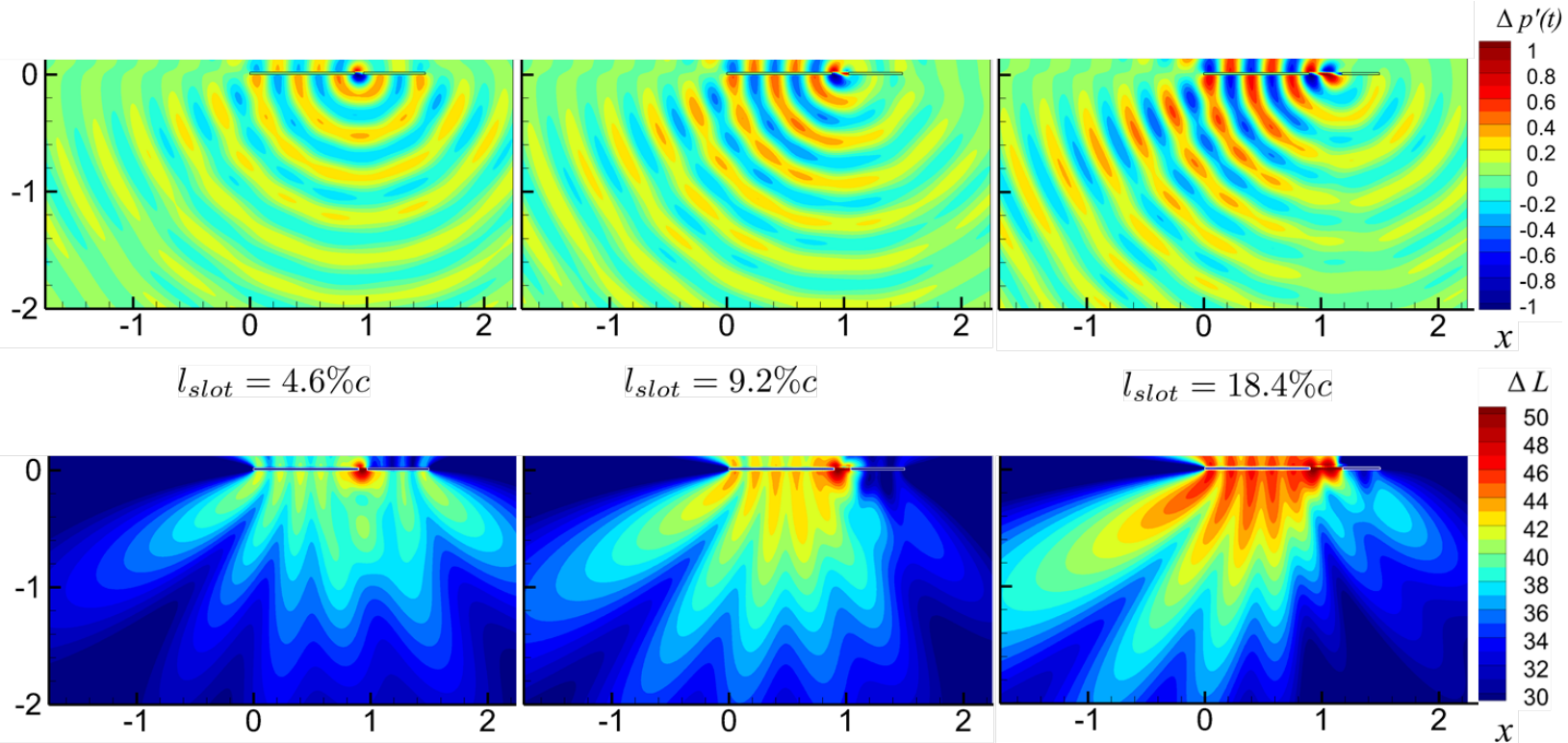

Figure 10. Difference pressure $\Delta p^{\prime}$ and level effect $\Delta L$ in the symmetry plane $z=0$ of the plate for three slotwidths at a distance $d=0.058 \mathrm{~m}$ of the shearlayer from the plate, $f=1000 \mathbf{H z}, \vartheta_{i}=\vartheta_{i}^{t o t}+2^{\circ}, M=0.3$.

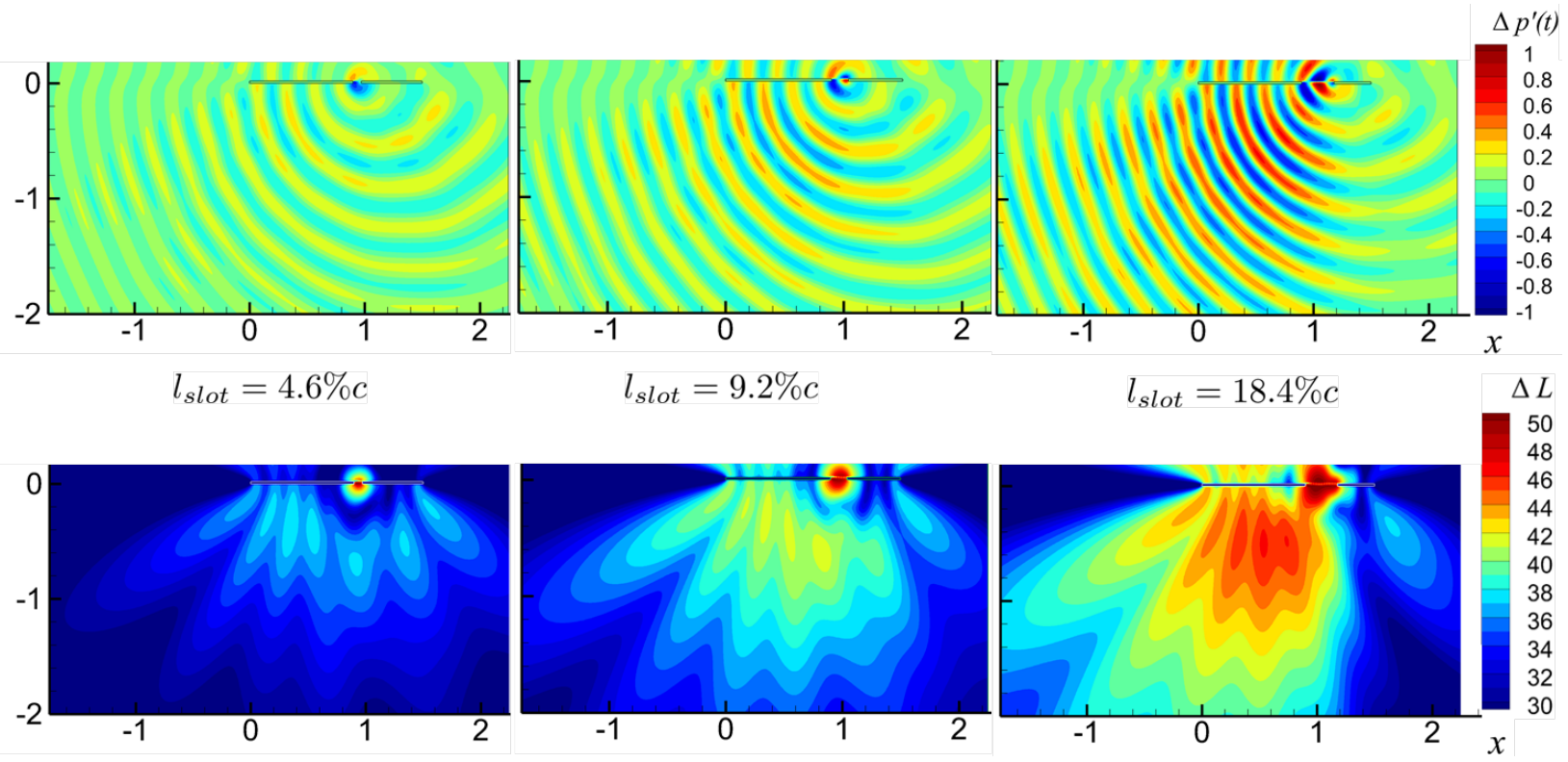

Figure 11. Difference pressure $\Delta p^{\prime}$ and level effect $\Delta L$ in the symmetry plane $z=0$ of the plate for three slotwidths and uniform flow speed $M=0.3, f=1000 \mathrm{~Hz}, \vartheta_{i}=\vartheta_{i}^{\text {tot }}+2^{\circ}, M=0.3$.

evanescent pressure fields below the shearlayer. Here, the only process leading to sound radiation from the slot region is related to the conversion of the existing evanescent field into sound. This effect to occur only requires a geometric inhomogeneity, which is given by the slot edges. It is noted that for the smallest slot width shown in figures 10 and 11 the shear induced field is stronger, while for the largest slot width the shear induced field is weaker than in the uniform flow case. It is quite plausible to generalize this finding to the problem of sound shielding at general geometries. Depending on the size of the slot/gap the neglect of shear in the flow field may either underestimate or overestimate the pressure level in the shadow zone.

Next, the influence of the proximity of the shearlayer to the plate is investigated. For this purpose the distance $d=0.058 \mathrm{~m}$, already shown in figure 10 was lowered to $d=0.03 \mathrm{~m}$ and finally $d=0.02 \mathrm{~m}$. This 

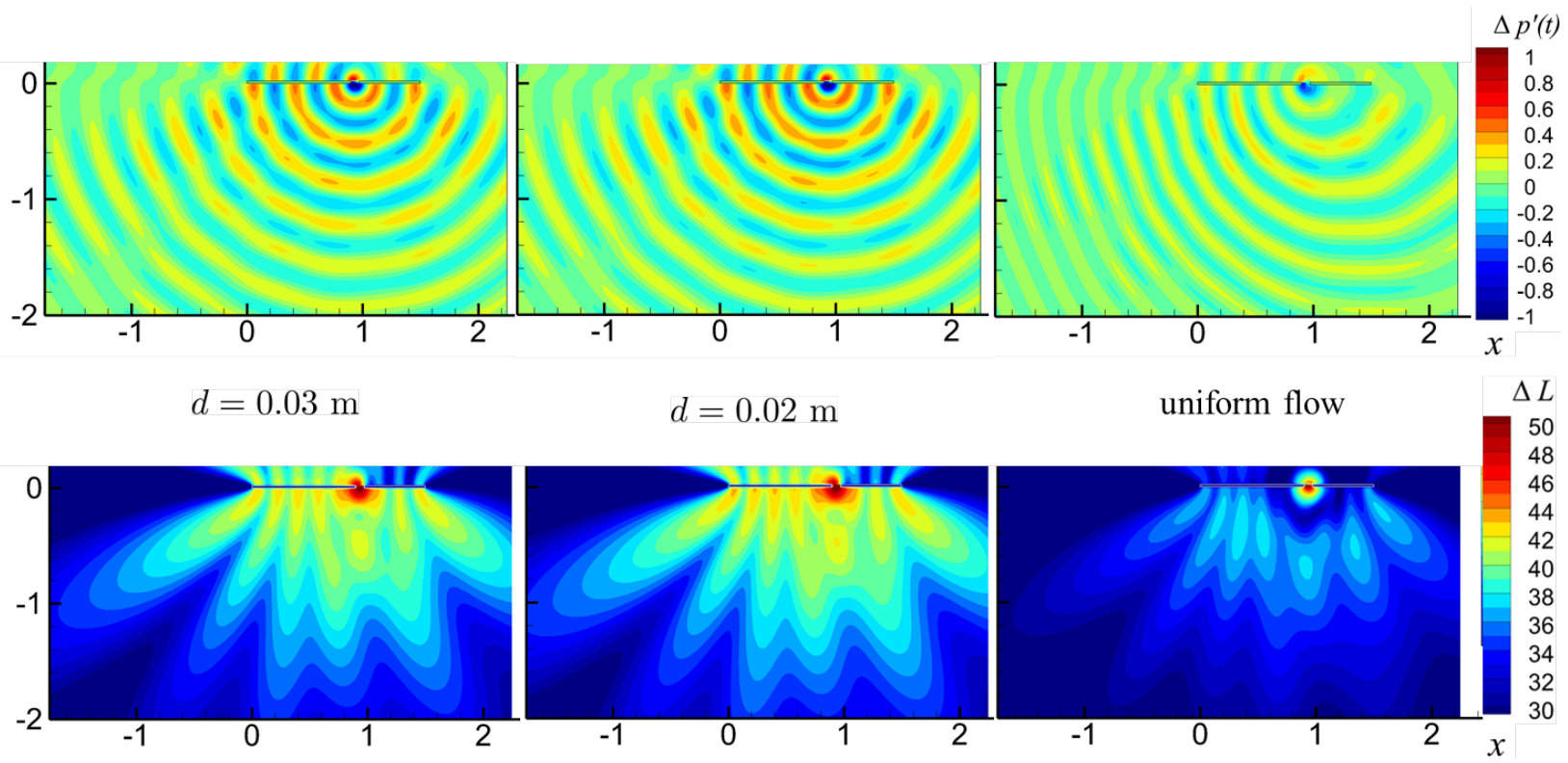

Figure 12. Difference pressure $\Delta p^{\prime}$ and level effect $\Delta L$ in the symmetry plane $z=0$ of the plate with slotwidth $l_{\text {slot }}=4.6 \% l_{c}$ and different distances $d$ of the shearlayer from the plate, $f=1000 \mathrm{~Hz}, \vartheta_{i}=\vartheta_{i}^{\text {tot }}+2^{\circ}, M=0.3$, right part: comparison to uniform flow case.

last distance represents a limiting case, since here the shearlayer touches the upper surface of the plate. The figure 12 depicts the effect, which a decrease of the distance has on the resulting sound field. One observes an increase of the signal amplitudes. This effect becomes more clear when comparing also the case for $d=0.058 \mathrm{~m}$. One may conclude from this observation that for a given flow Mach number the thinner the boundary layer (the distance $d$ being a representative parameter for it) the stronger the effect of it on the sound generation at the slot. This in turn may be interpreted as a Reynolds number dependence of the observed sound generation phenomena at the slot under the shearlayer. Obviously the shearlayer distance

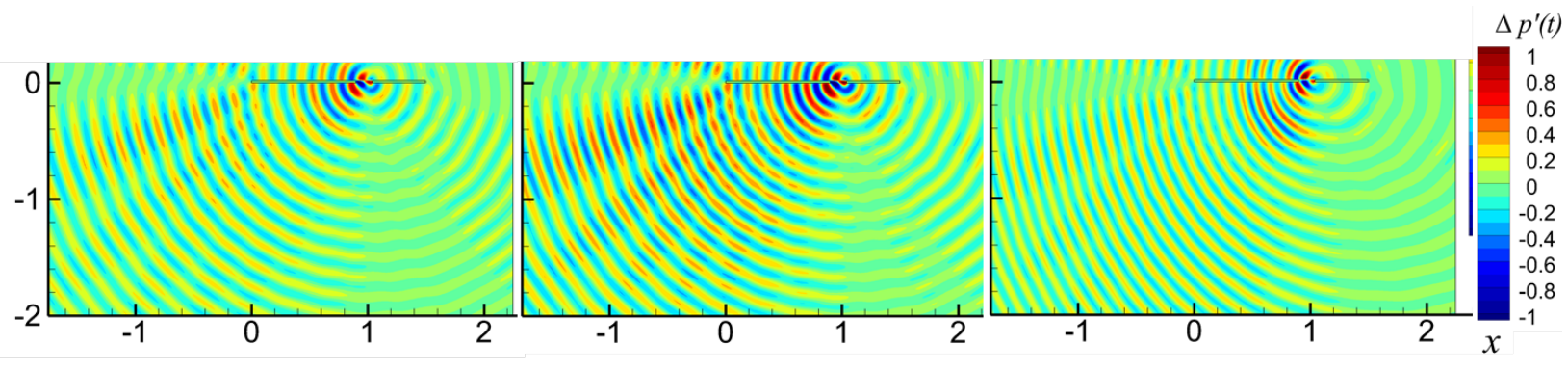

$d=0.058 \mathrm{~m}$

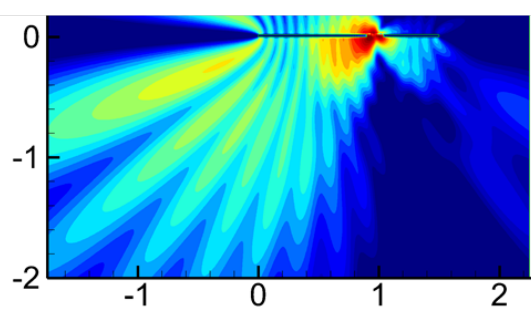

$d=0.03 \mathrm{~m}$

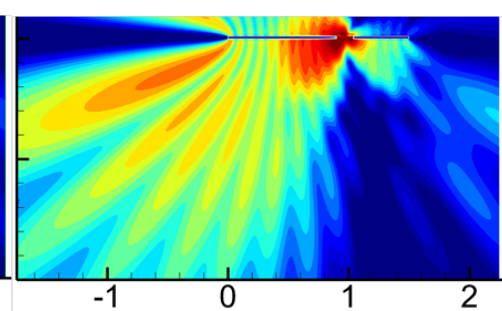

uniform flow

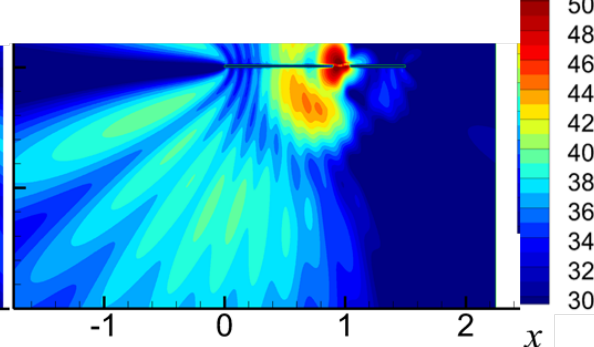

Figure 13. Difference pressure $\Delta p^{\prime}$ and level effect $\Delta L$ in the symmetry plane $z=0$ of the plate with slotwidth $l_{\text {slot }}=9.2 \% l_{c}$ and two different distances $d$ of the shearlayer from the plate, $f=2000 \mathbf{H z}, \vartheta_{i}=\vartheta_{i}^{t o t}+2^{\circ}, M=0.3$, right part: comparison to uniform flow case. 
is a parameter, which by definition has no influence on the case studied for uniform flow. As a reference, figure 12 also shows the field in this case, clearly indicating that the presence of the evanescent pressure fields beneath the shearlayer may lead to stronger sound fields at the slot compared to the uniform flow case.

The section on the slotted plate is closed with a look at the sound from the slot for an increased frequency. Figure 13 discusses again the effect of a change in distance of the shearlayer to the plate, but for the slot width $l_{\text {slot }}=9.2 \% l_{c}$ and for twice the frequency as before. The decrease of the distance shows a remarkable increase in pressure amplitudes. Again, the comparison with the case of uniform flow show consistently less sound emanating from the slot in this case too.

It is mentioned that further simulations showed that the incidence angle of the sound also slightly influence the sound field of the slot when the shear is present, while virtually no effect can be observed for the uniform flow. In this context it interesting that increasing the incidence angle further from the the value used throughout the study of the slotted plate by another $2^{\circ}$ resulted in a slight increase of the sound radiated from the slot.

\section{Shear flow effects at slot geometry - finite layer thickness}

The influence of a finite shear/boundary layer instead of an infinitely thin vortex sheet on the shielding problem is considered in this section. In order to isolate this effect from the influence of the leading and trailing edges, as well as the thickness of the plate, instead an infinitely extended slotted plate with zero thickness was investigated. The parameters were chosen similar to the original case shown in figure 4. The velocity profile $u^{0}$ along the ray, perpendicular to the suction side trailing edge point of the high lift system's main element was extracted and used as a parallel flow past the plate; it is non-dimensionalized with the speed of sound. Three different variants were computed, i) viscous parallel flow past the upper side of the plate and no flow below the plate (freestream Mach no. $M=0.3$, ii) the same viscous flow profile on both sides of the plate, and iii) uniform flow $M=0.3$ on both sides. The incident sound field comes from the same point source as in figure 4 with a frequency of $f=10 \mathrm{kHz}$ and unit strength $\hat{\theta}_{p}$; it is located at $(x, y)=(1.348,0.7) l_{c}$, where $l_{c}$ corresponds to the chord of the NACRE high lift system (see figure 4). The plate's vertical position is at $y=0.01448$. The slot's downstream edge is located such that it sees the source at an angle $2^{\circ}$ above the critical angle of total reflection, which for $M=0.3$ corresponds to the angle $\theta_{0}^{\text {tot }}=126.3^{\circ}$. This ensures that the entire slot is located in an area where no sound can directly penetrate through the shearlayer, i.e. the slot is subject to evanescent waves. The respective sound pressure levels

$$
L_{p}=20 \lg \left(p_{r m s}\right)+90
$$

are plotted in figure 14 wherein $p_{r m s}$ is non-dimensionalized with $\rho_{\infty} a_{\infty}^{2}$. The case without any geometry, i.e. the pure refraction of the shearlayer is also depicted in figure 21 for reference.

Since the source was introduced through a sponge layer extending from $y \simeq 0.45$ to the top end of the computation domain the free field of the isolated source is seen in this part of the plots. The distribution

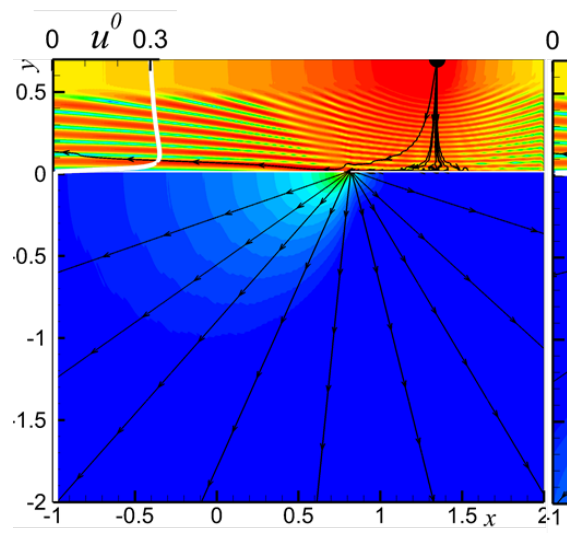

$0 \quad u^{0} 0.3$

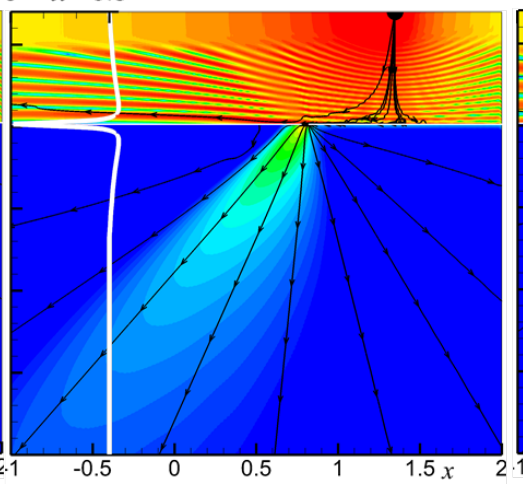

$0 \quad u^{0} 0.3$

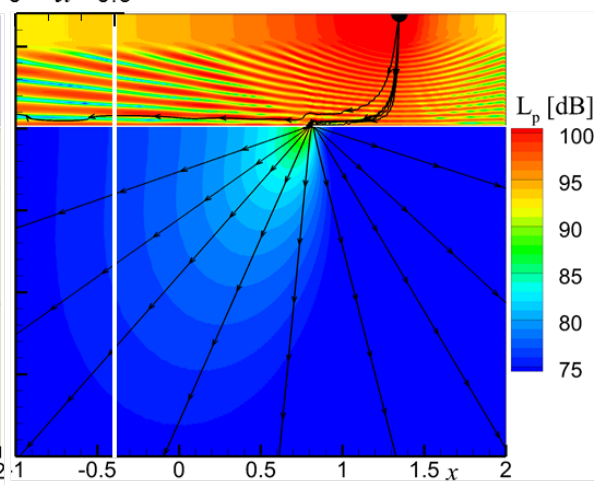

Figure 14. Sound pressure level $L_{p}$ for sound radiating through slot of width $l_{s l o t}=4.4 \% l_{c}$. Left: one-sided parallel flow, viscous velocity profile, center: two-sided parallel flow, viscous flow, right: uniform flow. The vector field lines indicate the direction of the sound intensity field. 
of isocontours clearly shows the convective amplification. The essentially horizontal strips in the half plane above the plate correspond to the pressure maxima of incident and reflected wave. The sound field radiated from the slot into the domain below the plate differs significantly for the three cases considered. As in the cases studied for the simple and slotted finite plate one generally observes an upstream oriented radiation pattern. While really the maximum sound radiation is seen in exactly the upstream direction for the case without flow below the plate (i), the refraction effect of the bottom side boundary layer causes this lobe to turn down and concentrate for case (ii). A simple radiation phenomenon is seen for the uniform flow case (iii), with a typical pattern for a convectively amplified simple source; see appendix B for a variation of the slotwidth. This case study shows that there is a strong concentrating and redirecting effect of the boundary layer on the lower side of the plate, which tends to increase the sound radiation induced by the evanescent waves below the boundary layer. In general one observes a non-neglidible flow effect on the radiation from the slot.

\section{Sound power considerations}

In the context of the relevance of evanescent waves beneath a boundary- or shearlayer on sound shielding it is interesting to investigate the sound intensity. The general question arises, whether or not sound power is generated when evanescent waves interact with an edge and thereby convert into propagational pressure signals. For this purpose the sound intensity was evaluated for one of the cases studied in the previous section on the slotted plate. Since the plate is immersed into non-moving medium (the flow exists only above the shearlayer, which in turn sits above the plate), the standard definition of sound intensity

$$
\boldsymbol{I}=\overline{p^{\prime} \boldsymbol{v}^{\prime}}=\frac{1}{2}\left(\hat{p}_{r} \hat{\boldsymbol{v}}_{r}+\hat{p}_{i} \hat{\boldsymbol{v}}_{i}\right)
$$

may be used, wherein $\boldsymbol{v}^{\prime}$ denotes the acoustic particle velocity vector and subscripts $r$ and $i$ denote real and imaginary part of the quantities, when expressed in the frequency domain (then denoted by a hat symbol). An evaluation of the sound intensity level defined as

$$
L_{I}=10 \lg |\boldsymbol{I}|+90
$$

is shown in figure 15, which also shows the vector field of the sound intensity. Several observations can be made. The sound intensity enters from the right (downstream side); all vectors are parallel to the flow; they are shown in the region of the evanescent waves, i.e. the evanescent waves do transport power. The presence of the plate causes the intensity vectors to deviate and spread to the lower half of the domain; interestingly, no sound power gets transmitted to the upper half. Since the wave field above the shearlayer is totally reflected, the sound power of the incident plane waves remains in the upper half. This suggests that indeed the interaction of the plate edges with the evanescent waves seems to generate propagational sound.

The study on the intensity was also carried out for the case of the shearlayer with finite thickness studied in section C. The results generated as a solution to the LEE are valid throughout the complete computaion

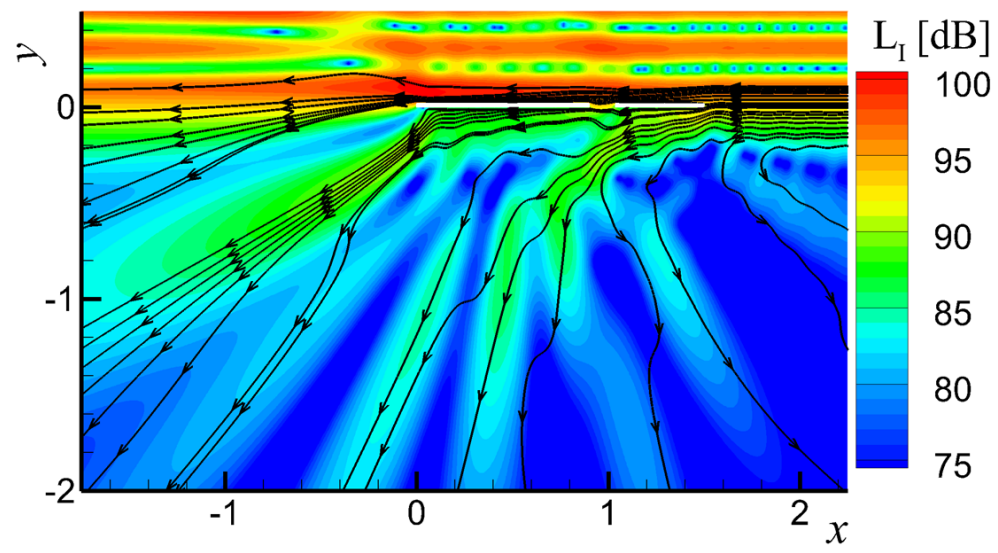

Figure 15. Sound intensity in the symmetry plane $z=0$ of the plate with slotwidth $l_{\text {slot }}=9.2 \% l_{c}, d=$ $0.058 \mathbf{m}, f=2000 \mathrm{~Hz}, \vartheta_{i}=\vartheta_{i}^{t o t}+2^{\circ}, M=0.3$, right part: comparison to uniform flow case. 

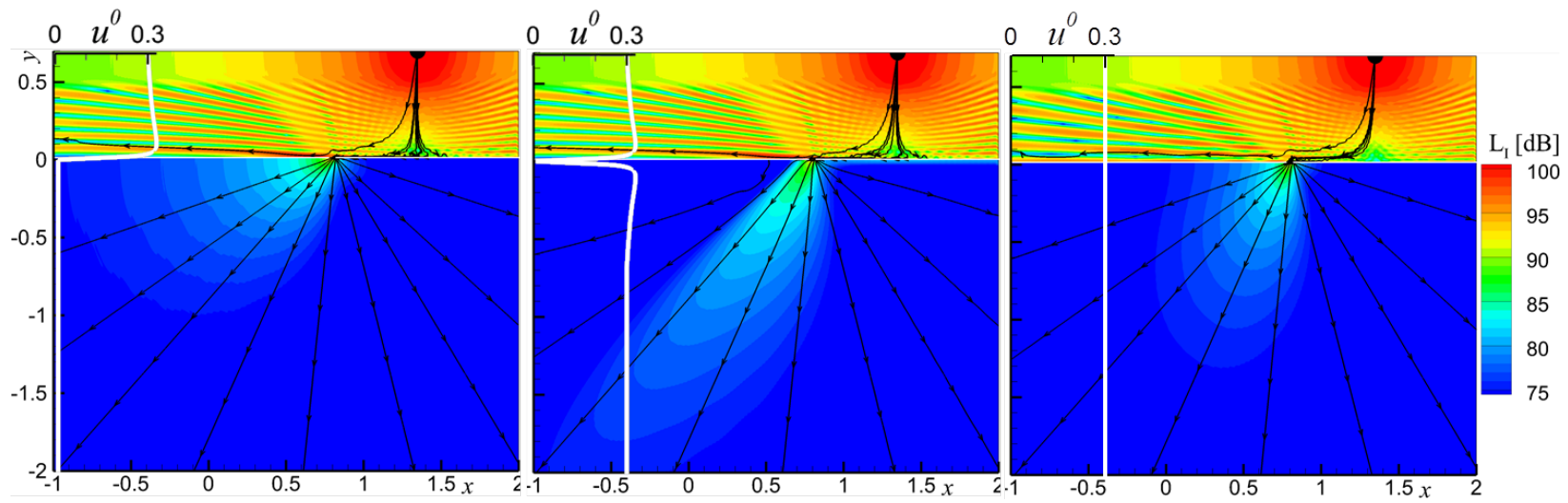

Figure 16. Sound intensity levell $L_{I}$ for sound radiating through slot of width $l_{s l o t}=4.4 \% l_{c}$. Left: one-sided parallel flow, viscous velocity profile, center: two-sided parallel flow, viscous flow, right: uniform flow. The vector field lines indicate the direction of the sound intensity field.

domain. Therefore the generalized intensity of Myers?

$$
\boldsymbol{I}=\overline{\left(p^{\prime}+\boldsymbol{M} \cdot \boldsymbol{v}^{\prime}\right)\left(\boldsymbol{v}^{\prime}+\boldsymbol{M} p^{\prime}\right)}=\frac{1}{2}\left[\left(\hat{p}_{r}+\boldsymbol{M} \cdot \hat{\boldsymbol{v}}_{r}\right)\left(\hat{\boldsymbol{v}}_{r}+\boldsymbol{M} \hat{p}_{r}\right)+\left(\hat{p}_{i}+\boldsymbol{M} \cdot \hat{\boldsymbol{v}}_{i}\right)\left(\hat{\boldsymbol{v}}_{i}+\boldsymbol{M} \hat{p}_{i}\right)\right]
$$

was evaluated along with the intensity vector field. Figure 16 shows the intensity level for the smallest slot width and the three different flow field as in figure 14. Except for the convective amplification the level is supposed to correspond to the sound pressure level in the far field. The natural absence of convective amplification in the intensity for the simple source used for this study is clearly seen in the level contours above the plate, which is in contrast to those seen for the pressure level in figure 16. One has to recall that Myers' definition of intensity 12 is stricly valid only for a potential background flow. Since a parallel shear flow is considered, the interpretion of $\boldsymbol{I}$ as a basis for the determination of the sound power

$$
P=\int_{\partial V} \boldsymbol{I} \cdot \boldsymbol{n} d A
$$
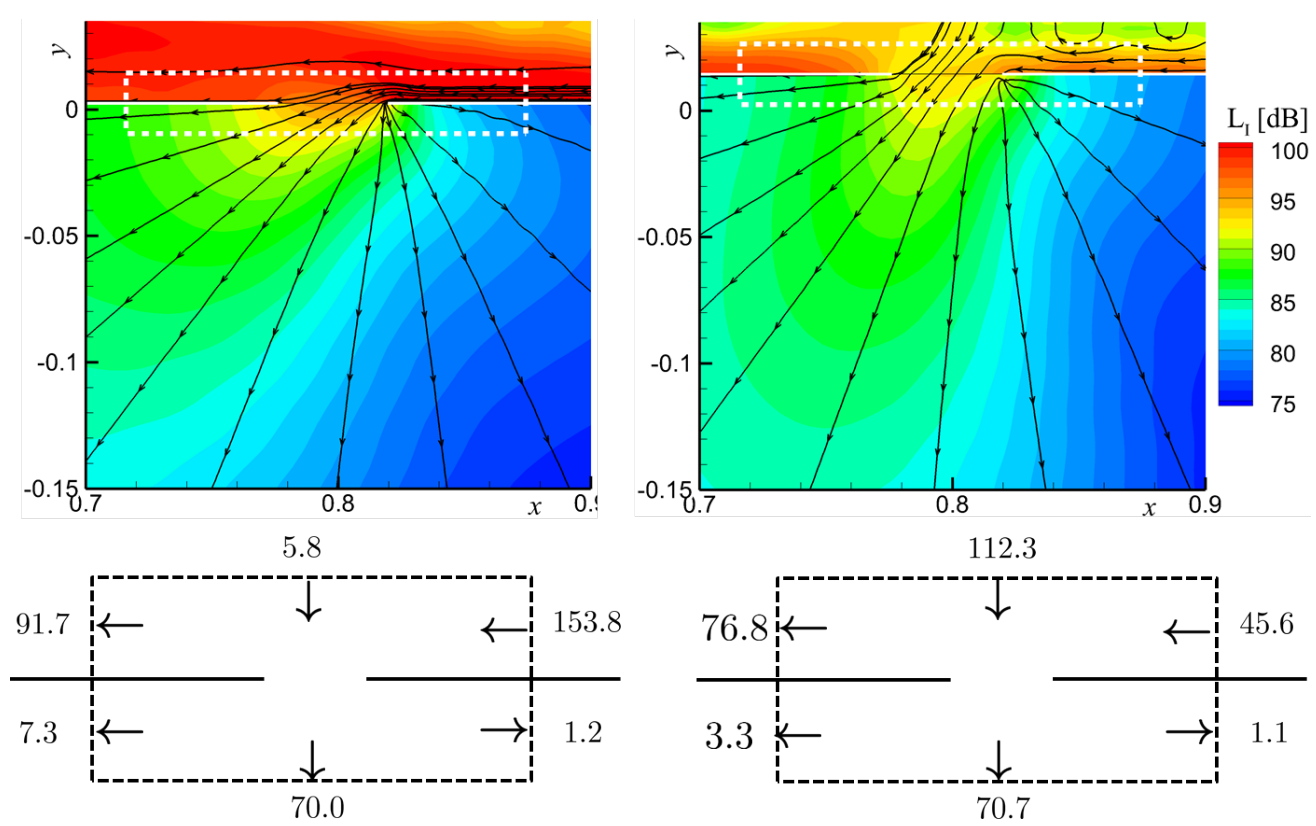

Figure 17. Sound power balance for box surrounding the slot of width $l_{\text {slot }}=4.4 \% l_{c}, \vartheta_{i}=\vartheta_{i}^{\text {tot }}+2^{\circ}$, left part: one sided parallel shear flow, right part: $M=0.3$ uniform flow. Values denote $|I \cdot n d A| \cdot 10^{3}$; arrows show the direction of flux.. 
is not simple. For this reason the case of the parallel shear flow past the upper side of the plate and no flow on the lower side (velocity profile see left part of 16) is re-considered. The plate was shifted slightly downwards by $1.2 \% l_{c}$, while the flow profile remained as before. The resulting intensity field is practically the same as before. However, the complete geometry is now immersed in a non-moving fluid, for which the standard definition 10 of the intensity may be used. In terms of a power balance this case is now compareable to the case of uniform flow (right part of figure 16 ), for which $\sqrt{12}$ ) is valid. The power balance was evaluated for a box containing the slot but extending only into areas, where either no flow or uniform flow is encountered. The box is shown in the two intensity plots presented on the upper part of figure 17. The lower part of the figure depicts the power flux through the sides of the respective box.

A look at the power flux through on the box above the plate shows a drastic difference between the two cases. For the shear flow case, almost all power enters through the thin downstream face of the box, representing the highly concentrated power contained in the evanescent waves. In contrast, for the case of uniform flow the split of incoming power is much more balanced between downstream and top face, clearly representing the incidence angle of the incoming wave. The vector field shown in the top of the figure confirms this view. It is quite interesting to observe that on the lower side of the box, both cases are quite compareable. This may be due to the fact that for the considered frequency the slot appears as a compact object.

The overall balance of the power flux over the box results in $P_{\text {uniform }}=-5.9 \cdot 10^{-3}$ for the uniform flow and $P_{\text {shear }}=+10.6 \cdot 10^{-3}$ for the shear flow. The question arises, why acoustic power is lost to the box in the uniform flow case. The computation is based on the LEE, implying theoretically no loss, the observed loss is therefore attributed to numerical dissipation of the CAA code. The main numerical influence is due to the near wall one-sided 7-stencil backward differencing scheme, which is combined with local damping operators. Since the length of the slot was resolved with 7 points, these operators become active throughout the entire slotwidth. The shear flow case on the other hand shows a net production of sound power, although the same numerical scheme on the same cartesian computation grid was used as for the uniform flow case. Assuming the same amount of numerical dissipation as in the uniform flow case one may conclude that the sound production is even underestimated for the shear flow situation.

Finally the net flux of acoustic power in/out the part of the box above and below the plate $\left(P_{\text {above }}\right.$ and $\left.P_{\text {below }}\right)$ is investigated separately. It is found that for the uniform flow case the net influx of acoustic power is $P_{\text {above }}=-81.0 \cdot 10^{-3}$, while $P_{\text {below }}=+75.1 \cdot 10^{-3}$ get radiated into the half plane below the slot. The balance evaluated when the shear flow is present results in $P_{\text {above }}=-67.9 \cdot 10^{-3}$, while $P_{\text {below }}=+78.5 \cdot 10^{-3}$. In summary, entirely different intensity fields are seen for the two cases considered, although the overall sound power radiated from the slot is pretty similar in value. There seems to be a net sound power production in the shear flow case, which might be explained by a phase shift between pressure and particle velocity of the evanescent field provoked by the edges of the slit. The simulations for the uniform flow revealed that there is a numerically induced power loss; the accompanying uncertainty may be reduced by simulations with higher resolution in the future.

\section{Conclusions}

- For the originally unexpected results of the numerical shielding study in ${ }^{5}$ an estimation confirmed that the conditions for the occurence of evanescent waves below the boundary layer over the high lift wings's upper surface in the vicinity of the flap slot are satisfied.

- A first analytical-numerical study of a simplified model problem with a plane shear layer and plane waves revealed that the evanescent waves are turned into propagational sound signals by means of an edge scattering mechanism. Thus there is a true sound generation process, rather than a simple spatial "reorientation" of the incident sound. The overall sound field originates from this incident plane wave.

- The signal undergoes a change from acoustic to non-acoustic and back to acoustic. In spite of a strong shearlayer total reflection, which by itself should provide a strong shielding effect, the sound manages to tunnel the zone below the shearlayer in the form of an evanescent wave field which partially gets turned back into sound by interaction with geometric inhomogeneities (here the gap slot with the respective edges) in the immediate vicinity of the shearlayer.

- This edge sound generation right in the flap slot radiates freely into the shadow zone and deteriorates the shielding effect predicted for the case of non-viscous flow, which lacks an evanescent wave mechansim. 

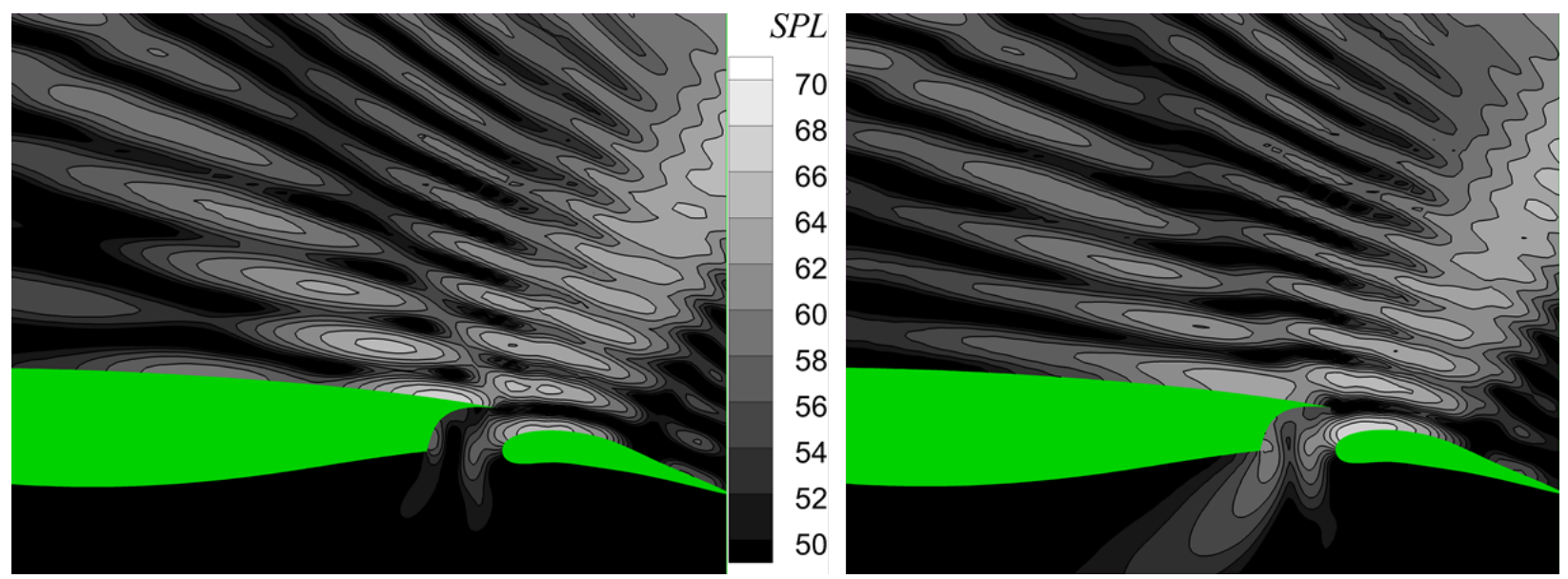

Figure 18. local pressure levels: left: constant flow, right: viscous flow, $f=11.733 \mathrm{kHz}$, chord length of retracted airfoil $c=0.543 \mathrm{~m}$.

The author believes that this tunnel effect, which is very similar to what is known as cuton/cutoff phenomena in duct problems, particularly the "zero splice liner problem", [6 satisfacorily explains the strong viscous effects observed in the shielding study of Dierke et al ${ }^{[5}$

One might think that the total reflection at the boundary layers would help the beneficial shielding effect. A considerable part of the shielding at the high lift system investigated by Dierke et a ${ }^{\sqrt{5}}$ actually happens at the boundary layer rather than at the surface of the airfoil. Figure 18 supports this observation because a comparison of the pressure rms values computed with constant flow (left part of figure) and those computed using the complete viscous flow shows characteristic differences near the wall. While the maximum of the rms pressure values naturally are located on the wall for the inviscid simulation ("pressure doubling at hard wall"), this maximum appears lifted upwards away from the wall for the viscous flow case. This strongly suggests that the pressure doubling (and hence the reflection) occurs above the wall in the shearlayer.

In spite of this beneficial effect of the total reflection it turns out that it deteriorates shielding when an edge or slot is present near the evanescent wave field below the shearlayer. At first sight it seems that the occurence of the sound source due to the tunnel effect would increase the overall sound power of the arrangement when compared to the result assuming inviscid flow. It is somewhat intriguing that the complete input sound power is totally reflected at the boundary layer, while additional sound is definitely produced at the edges in the region of the evanescent waves. Whether or not additional sound power is generated through this mechansim will be a highly interesing topic of further studies. The study suggests that the use of simplified CAA methods neglecting viscous mean flow effects for the predicion of sound shielding at aircraft geometries is save whenever the engine source is not located in the rear and at critical incidence on shearlayers in regions containing edges. For configurations differerent from that there may be the chance that beneficial shielding effects are broadly overpredicted by as much as $10 \mathrm{~dB}$ or so.

\section{Appendix}

\section{A. 2D point source}

For the generation of the sound source in the numerical solution of the LEE the 2D sound field of a point mass- or heat source in uniform flow was used: The acoustic field due to a harmonic line mass or heat source $\frac{D_{\infty} \dot{\theta}^{\prime}}{D t}$ with $\dot{\theta}^{\prime}=\hat{\theta}_{p} \exp (i \omega t) \delta\left(\boldsymbol{x}-\boldsymbol{\xi}_{0}\right)$ in two dimensions is

$$
\begin{aligned}
p^{\prime}(\boldsymbol{x}, t)=\frac{\omega \hat{\theta}_{p}}{4{\sqrt{1-M^{2}}}^{3}}\{ & {\left[J_{0}\left(k r^{*}\right)+\frac{M_{r}}{\sqrt{1-M^{2}+M_{r}^{2}}} Y_{1}\left(k r^{*}\right)\right] \cos \left(\omega t+k r \frac{M_{r}}{1-M^{2}}\right)+} \\
& {\left.\left[Y_{0}\left(k r^{*}\right)-\frac{M_{r}}{\sqrt{1-M^{2}+M_{r}^{2}}} J_{1}\left(k r^{*}\right)\right] \sin \left(\omega t+k r \frac{M_{r}}{1-M^{2}}\right)\right\} }
\end{aligned}
$$




$$
\begin{aligned}
\rho^{\prime}(\boldsymbol{x}, t)= & \frac{1}{a_{\infty}^{2}} p^{\prime}(\boldsymbol{x}, t) \\
\boldsymbol{v}^{\prime}(\boldsymbol{x}, t)= & \frac{-\omega \hat{\theta}_{p}}{4 a_{\infty} \rho_{\infty}{\sqrt{1-M^{2}}}^{3}}\left\{\left[\boldsymbol{M} J_{0}\left(k r^{*}\right)+\frac{\left(1-M^{2}\right) \boldsymbol{e}_{r}+M_{r} \boldsymbol{M}}{\sqrt{1-M^{2}+M_{r}^{2}}} Y_{1}\left(k r^{*}\right)\right] \cos \left(\omega t+k r \frac{M_{r}}{1-M^{2}}\right)+\right. \\
& \left.\quad\left[\boldsymbol{M} Y_{0}\left(k r^{*}\right)-\frac{\left(1-M^{2}\right) \boldsymbol{e}_{r}+M_{r} \boldsymbol{M}}{\sqrt{1-M^{2}+M_{r}^{2}}} J_{1}\left(k r^{*}\right)\right] \sin \left(\omega t+k r \frac{M_{r}}{1-M^{2}}\right)\right\}
\end{aligned}
$$

where $r^{*}=r \sqrt{1-M^{2}+M_{r}^{2}} /\left(1-M^{2}\right)$, in which $r=|\boldsymbol{r}|$ with $\boldsymbol{r}=\boldsymbol{x}-\boldsymbol{\xi}_{0}$ and $M_{r}=\boldsymbol{r} \cdot \boldsymbol{M} / r$ while $\boldsymbol{e}_{r}=\boldsymbol{r} / r$.

\section{B. Influence of slot width on sound radiation for finite shearlayers}

In this section further simulation results are given concerning the effect of a realistic shearlayer for different slotwidths. In addition to figure 14 figure 19 depicts this influence on the sound pressure level $L_{p}$ for a slotwidth of $7.1 \% l_{c}$ and $21.3 \% l_{c}$. The corresponding intensity fields are plotted in figure 20. Note the

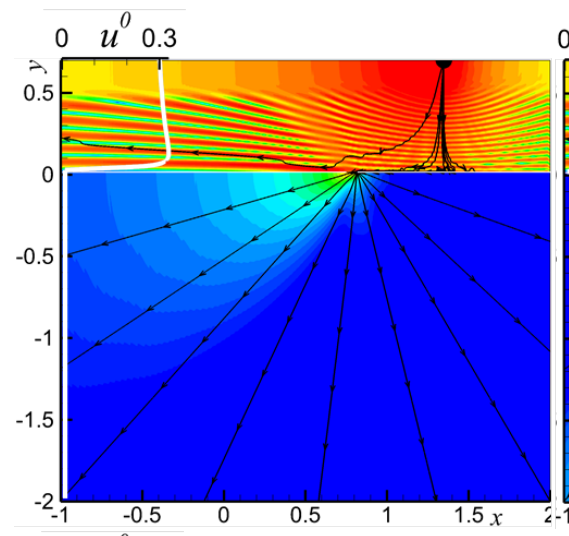

$0 \quad u^{0} 0.3$

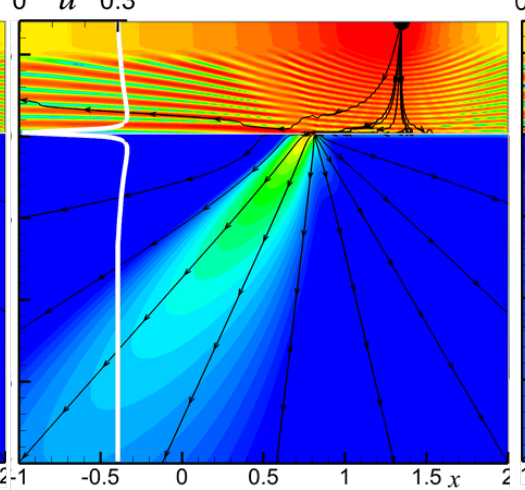

$0 \quad u^{0} 0.3$

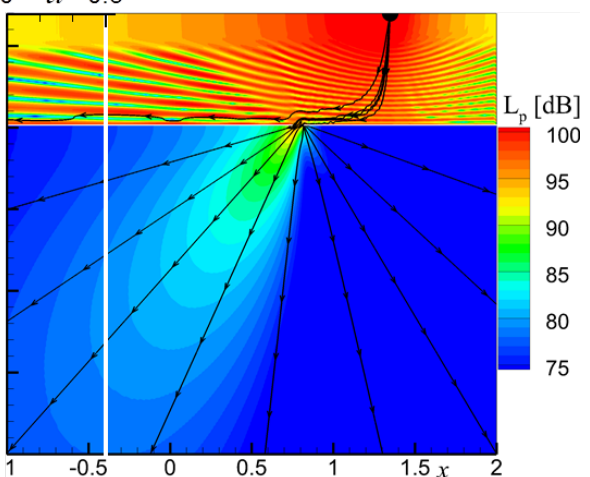

$0 \quad u^{0} 0.3$

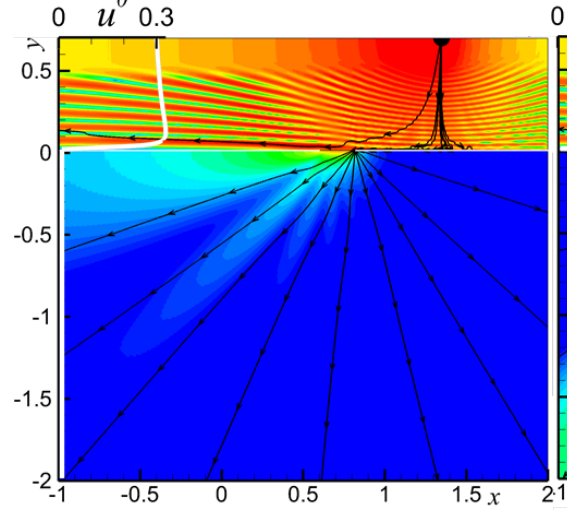

$0 \quad u^{0} 0.3$
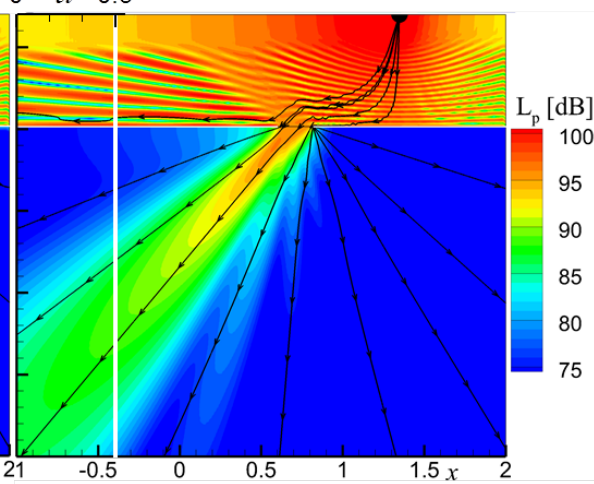

Figure 19. Sound pressure level $L_{p}$ for sound radiating through slot of width $l_{\text {slot }}=7.1 \% l_{c}$ (upper row) and $l_{\text {slot }}=21.3 \% l_{c}$ (lower row). Left: one-sided parallel flow, viscous velocity profile, center: two-sided parallel flow, viscous flow, right: uniform flow. The vector field lines indicate the direction of the sound intensity field.

difference between the sound pressure level $L_{p}$ and the sound intensity level $L_{I}$ due to the convective amplication.

\section{Acknowledgments}

The author likes to thank F. Quandt for the numerical simulation done for the solution of the Linearized Euler Equations. The author also likes to thank Dr. M. Lummer for providing reference solutions from DLR's Fast Multipole BEM Code FMBEM. Finally, the author acknowledges very helpful discussions with Prof. S.K. Lele on the topic dealt with in the paper. 

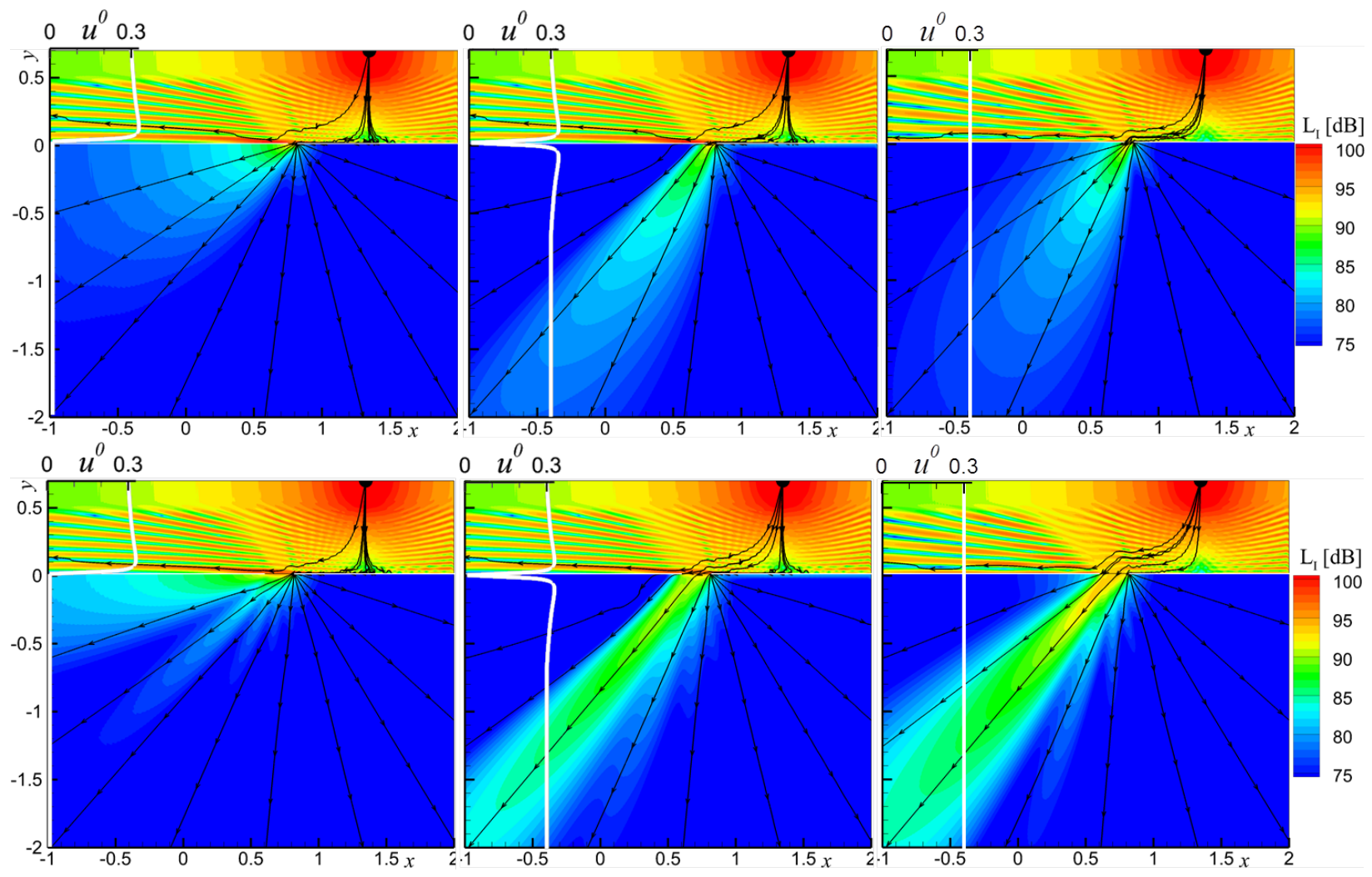

Figure 20. Sound intensity level $L_{I}$ and vector field for sound radiating through slot of width $l_{s l o t}=7.1 \% l_{c}$ (upper row) and $l_{\text {slot }}=21.3 \% l_{c}$ (lower row). Left: one-sided parallel flow, viscous velocity profile, center: two-sided parallel flow, viscous flow, right: uniform flow. The vector field lines indicate the direction of the sound intensity field.
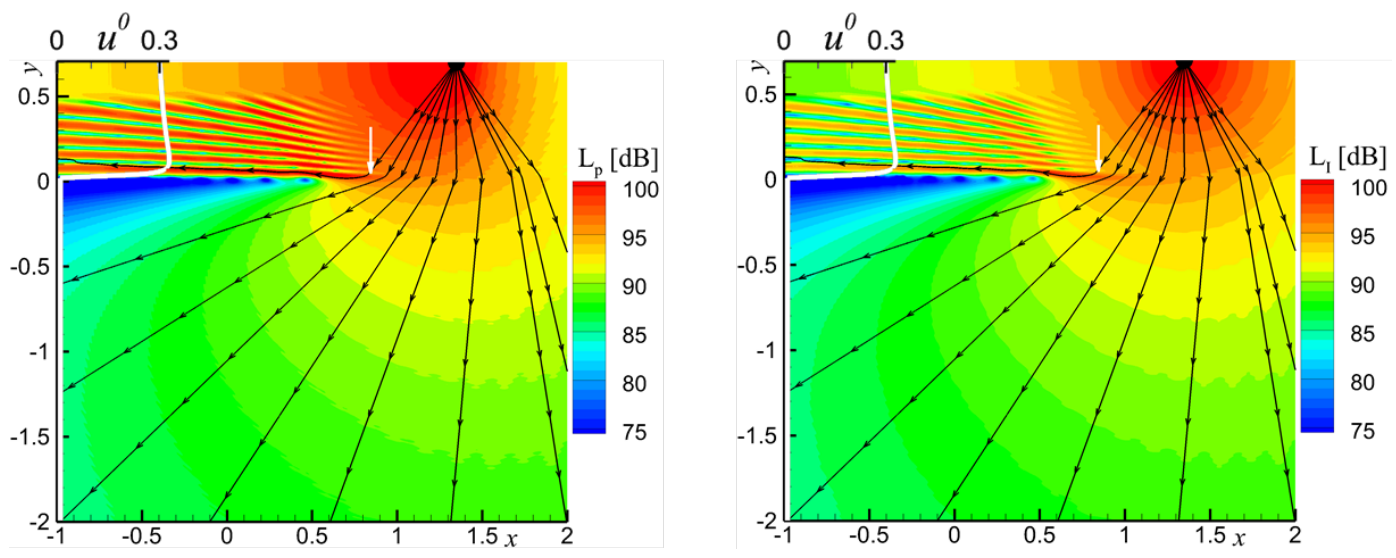

Figure 21. Sound pressure level $L_{p}$ and sound intensity level $L_{I}$ and intensity vector field for sound radiation of point source in the presence of a shearlayer. The white arrow indicates the location of the point of total reflection $(x, y)_{t o t}=(0.8446,0.0144) l_{c}$ for a uniform flow of $M=0.3$ in the half plane above $y=0.01448 l_{c}$.

\section{References}

${ }^{1}$ Astley, R. J.; Bain, J. G.: A Three-Dimensional Boundary Element Scheme for Acoustic Radiation in Low Mach Number Flows, Journal of Sound and Vibration, Vol. 109, 1986, pp. 445465.

${ }^{2}$ Clancy, C.; Rice, H.J.: Acoustic shielding in low mach number potential flow incorporating a wake model using BEM. AIAA Paper 2009-3174, 2009

${ }^{3}$ Delfs, J. W.; Bauer, M.; Ewert, R.; Grogger, H. A.; Lummer, M.; and Lauke; T. G. W., Numerical Simulation of Aerodynamic Noise with DLR's aeroacoustic code PIANO," Handbook 2008. 
${ }^{4}$ Delfs, J.: lecture notes "Basics of Aeroacoustics" (in english), http://www.dlr.de/as/desktopdefault.aspx/ tabid-191/401_read-22566/skript_GrundlagenAeroakustikWS.pdf

${ }^{5}$ Dierke, J.; Ewert, R., Chappuis, J.; Lidoine, S.; Ricouard, J: The influence of realisitc 3D viscous mean flow on shielding of engine-fan noise by a 3-element high lift wing. AIAA 2010-3917, 16th AIAA/CEAS Aeroacoustics Conference 2010.

${ }^{6}$ Fuller, C.R.: Propagation and Radiation of Sound from Flanged Circular Ducts with Circumferentially Varying Wall Admittances, I.: Semi-Infinite Ducts, Journal of Sound and Vibration, Vol. 93, No. 3, 1984, pp. 321340.

${ }^{7}$ Haibel, A.; Nimtz, G.; Stahlhofen, A.A.: Frustrated total reflection: the double-prism revisited. Physical Review E, Vol. 63, 047601, 2001.

${ }^{8}$ Myers, M.K.: Generalization and Extension of the Law of Acoustic Energy Conservation in a Nonuniform flow. AIAA 86-0471.

${ }^{9}$ Tinetti, A. F.; Dunn, M. V.: Aeroacoustic Noise Prediction Using the Fast Scattering Code, Proceedings of the 11th AIAA/CEAS Aeroacoustics Conference, AIAA Paper 2005-3061, 2005, pp. 112.

${ }^{10}$ Wolf, W.R.; Lele, S.K.: Fast Acoustic Scattering Simulations with Non-Uniform Potential Flow Effects. 